\title{
Metagenomic analysis of gut microbiome and resistome of diarrheal fecal samples from Kolkata, India, reveals the core and variable microbiota including signatures of microbial dark matter
}

\author{
Rituparna De* ${ }^{*}$, Asish Kumar Mukhopadhyay and Shanta Dutta
}

\begin{abstract}
Background: Metagenomic analysis of the gut microbiome and resistome is instrumental for understanding the dynamics of diarrheal pathogenesis and antimicrobial resistance transmission (AMR). Metagenomic sequencing of 20 diarrheal fecal samples from Kolkata was conducted to understand the core and variable gut microbiota. Five of these samples were used for resistome analysis. The pilot study was conducted to determine a microbiota signature and the source of antimicrobial resistance genes (ARGs) in the diarrheal gut.

Results: $16 \mathrm{~S}$ rRNA amplicon sequencing was performed using Illumina MiSeq platform and analysed using the MGnify pipeline. The Genome Taxonomy Database (GTDB-Tk) was used for bacterial taxonomic identification. Diarrheal etiology was determined by culture method. Phylum Firmicutes, Bacteroidetes, Proteobacteria and Actinobacteria were consistently present in 20 samples. Firmicutes was the most abundant phylum in 11 samples. The Bacteroidetes/Firmicutes ratio was less than 1 in 18 samples. 584 genera were observed. 18 of these were present in all the 20 samples. Proteobacteria was the dominant phylum in 6 samples associated with Vibrio cholerae infection. Conservation of operational taxonomic units (OTUs) among all the samples indicated the existence of a core microbiome. Asymptomatic carriage of pathogens like Vibrio cholerae and Helicobacter pylori was found. Signature of Candidate phyla or "microbial dark matter" occurred. Significant correlation of relative abundance of bacterial families of commensals and pathogens were found. Whole-genome sequencing (WGS) on Illumina MiSeq system and assembly of raw reads using metaSPAdes v3.9.1 was performed to study the resistome of 5 samples. ABRicate was used to assign ARG function. 491 resistance determinants were identified. In $80 \%$ of the samples tetracycline resistance was the most abundant resistance determinant. High abundance of ARGs against $\beta$-lactams, aminoglycosides, quinolones and macrolides was found. Eschericia sp. was the major contributor of ARGs.

Conclusions: This is the first comparative study of the gut microbiome associated with different diarrheal pathogens. It presents the first catalogue of different bacterial taxa representing the core and variable microbiome in acute diarrheal patients. The study helped to define a trend in the gut microbiota signature associated with diarrhea and revealed which ARGs are abundantly present and the metagenome-assembled genomes (MAGs) contributing to AMR.
\end{abstract}

*Correspondence: rituparna26@gmail.com

Division of Bacteriology, National Institute of Cholera and Enteric

Diseases, Kolkata, India

(c) The Author(s) 2020. This article is licensed under a Creative Commons Attribution 4.0 International License, which permits use, sharing, adaptation, distribution and reproduction in any medium or format, as long as you give appropriate credit to the original author(s) and the source, provide a link to the Creative Commons licence, and indicate if changes were made. The images or other third party material in this article are included in the article's Creative Commons licence, unless indicated otherwise in a credit line to the material. If material is not included in the article's Creative Commons licence and your intended use is not permitted by statutory regulation or exceeds the permitted use, you will need to obtain permission directly from the copyright holder. To view a copy of this licence, visit http://creativeco mmons.org/licenses/by/4.0/. The Creative Commons Public Domain Dedication waiver (http://creativecommons.org/publicdomain/ zero/1.0/) applies to the data made available in this article, unless otherwise stated in a credit line to the data. 


\section{Background}

Diarrhea is a leading cause of mortality accounting for more than 1.6 million deaths worldwide [1]. It causes nearly 5,25,000 deaths among children under 5 years of age and leads to malnutrition, stunted growth and anemia [2-7]. It is particularly prevalent in the low and middle-income countries owing to poor hygiene and sanitation. India is the second most populous country in the world and is one of the top five countries with the highest burden of diarrhea and high rates of mortality and morbidity [8-10]. Recently, India has recorded the highest number of deaths among under five age group [11]. The Eastern region recorded the third highest mortality rate among under five age and diarrhea is one of the leading causes of death in this region [11]. In India the most common causes of diarrhea are Rotavirus, Cryptosporidium sp. Shigella sp., Enterotoxigenic Eschericia coli $[12,16]$. Antibiotic therapy is administered to diarrheal patients along with ORS (oral rehydration solution) to assuage severity of symptoms. AMR (antimicrobial resistance) has rendered antibiotic therapy in diarrhea partially or completely ineffective. The genetic determinants of AMR reside in the gut and in the environmental microbiota from where they spread and enter into diarrheal pathogens by lateral gene transfer (LGT). Most of the diarrheal pathogens like E.coli, Klebsiella pneumoniae, Campylobacter sp., Shigella sp. have emerged as multidrug-resistant (MDR) and extensively drug-resistant (XDR) and fail to respond to empirical drugs like aminoglycosides and cephalosporin [13]. AMR is a global challenge which needs to be urgently addressed using a multi-disciplinary approach. Surveillance of AMR in diarrheal patients based on next-generation sequencing (NGS) is a novel way of addressing the AMR threat [13]. The structural and functional components of the microbiota can be studied and mapped completely with the aid of culture-free techniques which have been possible due to the advent of NGS. Big data derived from sequencing metagenomes will help to understand the importance of the structural and functional components of the microbiota in the development and dissemination of AMR [13] by detection, analysis of distribution and abundance of AMR determinants and their source organisms. A large number of studies have been undertaken over the last decades to understand the human microbiome and its association with disease $[14,15]$. A lot of emphasis has been put on defining a healthy microbiome signature and core microbiome culminating in the Human Microbiome Project for cataloguing the microbial communities in different body sites. These projects have revealed that the gut microbiome is one of the most diverse and complex [14, 16-18]. Although a core microbiome may exist every individual has a unique microbiota which is shaped by various parameters like genetic make-up, ethnicity, altitude, geographical location, mode of delivery and diet among others and also changes with age, travel, exposure to antibiotics and infections $[14,15,19-22]$ and onset of diseases [23].

The microbiota comprises archae, bacteria, viruses and unicellular eukaryotes. These carry out essential functions which are indispensible for maintaining a healthy state of the body and includes homeostasis, metabolism, immunity. This symbiotic association between the host and the microbiota is highly vulnerable as the fragile structure of the microbiota is prone to dysbiosis in the event of diseases. In the disease state the commensal flora is subdued by pathobionts (opportunistic pathogens and asymptomatically carried pathogens) [24]. The most common observation is Bacteroidetes/Firmicutes ratio which is high in the healthy state is reversed in the disease state with few exceptions [25]. Dysbiosis has been frequently studied in metabolic disorders [26], cancer [27], inflammatory diseases [28]. Specific microbes and specific signature of gut microbiota termed as enterotypes have been found to be associated with each of the diseases [29]. Only few studies have addressed gut microbiota dysbiosis in diarrhea. Most of these studies have been directed towards understanding dysbiosis in the event of infection by individual pathogens [30-33] or in hospital acquired infections (HAIs) [34] or in Traveler's diarrhea (TD) [25].

The current study is an unbiased pilot study conducted for characterizing the gut microbiota and the resistome from diarrheal stool and to see if we could find a statistically significant association of microbiota structure with diarrhea. We present the first comparative analysis of gut microbiota from twenty fecal samples collected from patients with symptoms of diarrhea. The stool samples were collected at the Infectious Diseases Beliaghata General Hospital (IDH) and Dr. B.C. Roy Memorial Hospital for Children $(\mathrm{BCH})$, both in Kolkata, in Eastern India. These were subject to diagnostic test by classical microbiological method and were found to be associated with either distinct diarrheal etiology or with mixed infections and for some the etiology could not be determined by culture method currently deployed in our laboratory. Eastern India is endemic for diarrhea. Kolkata is a cosmopolitan city with a population of 5.8 million. It is the capital of the state of West Bengal (Fig. 1) and a major commercial hub of India where people of high, middle and low-income groups throng for job and business opportunities from across the country contributing to the remarkable cultural and ethnic diversity of the city. The Infectious Diseases and B.C. Roy Memorial Hospital in Kolkata has specialized facility for the treatment of diarrheal patients. It is the apex referral centre and sentinel 


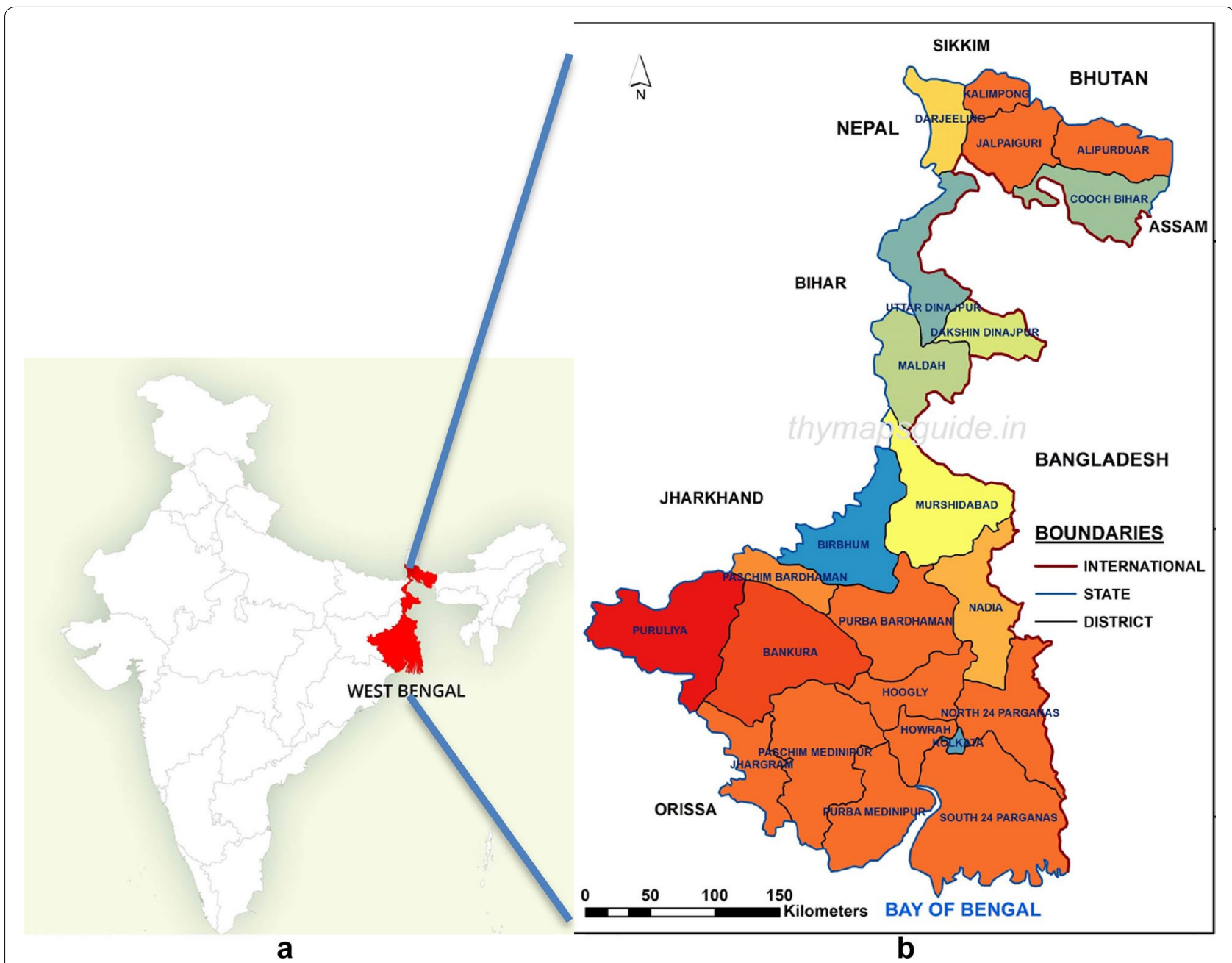

Fig. 1 Showing West Bengal in Eastern India (Courtesy: thymapguide.in)

surveillance centre for infectious diseases in West Bengal and Eastern India. Regular diarrheal stool collection takes place from the outpatient ward and from hospitalized patients. Therefore, NGS applied to study diversity of bacterial composition of the gut microbiome is anticipated to reveal striking biodiversity. The results could be a valuable resource for understanding the gut microbiota composition and resistome in the region. In our study we present the profile of the gut microbiota using $16 \mathrm{~S}$ rRNA amplicon sequencing and resistome using whole genome shotgun (WGS) sequencing in diarrheal patients who were not subjected to any selective bias. They were randomly selected to represent the heterogeneity in a real community to catalogue the diversity of bacterial species present in the gut microbiota of the local community and in spite of observed inter-individual differences in enterotypes to define a shared microbiome. The study helped to understand the importance of the composition of the diarrheal gut microbiota that may be contributing to diarrheal pathogenesis, AMR and identify organisms that may be exploited to counterfeit the effect of diarrhea. The study helped to establish a catalogue of taxonomic units present in the gut microbiome of diarrheal subjects and to understand the superiority of WGS over $16 \mathrm{~S}$ amplicon sequencing in studying the structure of the microbiota.

\section{Results}

Demographic details and diagnosis of fecal specimen Out of 20 diarrheal fecal samples 13 were from male patients and 7 were from females. The cohort included subjects from the age of 8 months to 56 years which were divided into three, age groups namely, 0-5 years, 6-15 years and above 15 years. Accordingly, 5 samples could be assigned to $0-5$ years group, 2 samples were assigned to $6-15$ years group and 13 samples were assigned to above 15 years group. S1, S2, S4, S16 and S17 
were from the outpatient ward while the remaining samples were collected from hospitalized diarrheal patients.

Diagnosis of diarrheal pathogen by culture-based methods showed that S1, S2, S5, S11, S12, S14, S18, S20 were associated with Vibrio cholerae (VC) O1; S14 with VC O139; S4, S7 with VC non O1 non O139; S19 with Vibrio fluvialis; S15 and S16 with Aeromonas sp.; S3, S6, S8, S9 suffered mixed infections; S17 with Shigella flexeneri; the diarrheal pathogen associated with S10 could not be determined with culture method established in our laboratory.

Diarrheal study subjects' demographic details and culture results have been presented in Table 1 .

\section{S rDNA V3-V4 amplicon sequencing}

\section{Gut microbiota of diarrheal patients}

$16 \mathrm{~S}$ rDNA sequencing was carried out to study structural composition of diarrheal microbiome and the relative abundance of various components of the microbiota. $16 \mathrm{~S}$ rDNAV3-V4 sequencing of the diarrheal samples (Fig. 2) yielded $>150 \mathrm{~K}$ raw reads per sample. Of these $88 \%-91 \%$ passed quality control. These processed reads ranged in size from 100 to $478 \mathrm{bp}$ with an average sequence size of 200-300 bp for each sample.
The samples uniformly showed the presence of Superkingdom (SK) Bacteria as the major constituent of the diarrheal microbiota in every sample. SK Chloroplast was also found but in minute proportion compared to Bacteria. SKs Archae, Mitochondria and Eukaryota also appeared in minute proportion in many of the samples but not all.

Histograms representing the relative abundance of different phyla, class, order, family, genera and species were constructed with $0 \%$ threshold and occur in Fig. 3. A total of 46 bacterial phyla were found by DNA sequence homology to reference genomes on the GTDB-Tk database. Bacterial phyla that were present in all the twenty samples were Firmicutes, Bacteroidetes, Actinobacteria and Proteobacteria. Firmicutes was the most dominant phylum in S3 (58.01\%), S5 (44.97\%), S7 (77.26\%), S10 (51.81\%), S11 (41.21\%), S12 (37.64\%), S14 (67.07\%), S16 (75.69\%), S17 (54.03\%), S19 (40.16\%), S20 (62.89\%) irrespective of the diarrheal pathogen that was isolated from it followed by Proteobacteria which was the most dominant phylum in S1 (46.27\%), S2 (25.01\%), S4 (35.54\%), S6 (38.91\%), S8 (14.1\%), S18 (63.09\%). Actinobacteria was the most dominant in $\mathrm{S} 9$ with $49.58 \%$ abundance rate followed by $47.82 \%$ of Firmicutes. Actinobacteria was the most abundant in S15 with $42.76 \%$ followed by $31.82 \%$

Table 1 Demographic details of the donors of diarrheal stool, the pathogen isolated, the most abundant phylum and Bacteroidetes/Firmicutes (B/F) ratio

\begin{tabular}{|c|c|c|c|c|c|c|c|}
\hline Sample ID & Sex & $\begin{array}{l}\text { Age (Years } \\
\text { (y)/Months } \\
\text { (m)) }\end{array}$ & $\begin{array}{l}\text { Hospitalized } \\
\text { (H)/OPD(O) }\end{array}$ & District/State & Pathogen isolated by culture & Most abundant phylum & $\mathrm{B} / \mathrm{F}$ ratio \\
\hline S1 & Male & $29 y$ & $\mathrm{O}$ & 24 Parganas & VCO1 Inaba & Proteobacteria & 0.256637168 \\
\hline S2 & Female & $52 y$ & O & 24 Parganas & VCO1 Ogawa & Proteobacteria & 0.788744975 \\
\hline S3 & Male & $36 y$ & H (3 days) & Kolkata & EAEC,VC O1 Ogawa & Firmicutes & 0.00844682 \\
\hline S4 & Male & $2 y$ & $\mathrm{O}$ & 24 Parganas & VCNon-O1 nonO139 & Proteobacteria & 0.042666667 \\
\hline S5 & Male & $11 y$ & H (2 days) & 24 Parganas & VCO1 Ogawa & Firmicutes & 0.659106071 \\
\hline S6 & Female & $25 y$ & H (1 day) & Kolkata & VCO1 Ogawa + E.coli (ETEC LTST) & Proteobacteria & 0.105140187 \\
\hline S7 & Female & $43 y$ & H (1 day) & Kolkata & VC Non-O1 nonO139 & Firmicutes & 0.02601605 \\
\hline S8 & Male & $22 y$ & H (3 days) & Burdwan & VCO1 Inaba+Campylobacter sp. & Proteobacteria & 0.901763224 \\
\hline S9 & Male & $16 y$ & H (2 days) & Kolkata & VCO1 Ogawa + C.jejuni & Actinobacteria & 0.02446675 \\
\hline S10 & Male & $55 y$ & H (1 day) & Kolkata & UNRESOLVED & Firmicutes & 0.119475005 \\
\hline S11 & Female & $56 y$ & H (1 day) & Kolkata & VCO1 Ogawa & Firmicutes & 0.01601553 \\
\hline S12 & Male & $12 y$ & H (1 day) & Kolkata & VCO1 Ogawa & Firmicutes & 0.681455898 \\
\hline S13 & Male & $40 y$ & H (3 days) & Kolkata & VCO139 & Bacteroidetes & 1.157114228 \\
\hline S14 & Male & $50 y$ & H (1 day) & Hooghly & VCO1 Ogawa & Firmicutes & 0.023855673 \\
\hline S15 & Male & $1 y$ & H (1 day) & Kolkata & Aeromonas sp. & Actinobacteria & 1.536455818 \\
\hline S16 & Female & $8 m$ & $\mathrm{O}$ & Kolkata & Aeromonas sp. & Firmicutes & 0.001056943 \\
\hline S17 & Female & $2 y$ & $\mathrm{O}$ & Kolkata & S.flexeneri (UT) & Firmicutes & 0.152137701 \\
\hline S18 & Male & $35 y$ & H (2 days) & Bihar & VCO1 Ogawa & Proteobacteria & 0.864882507 \\
\hline S19 & Female & $4 y$ & H (1 day) & Kolkata & V.fluvialis & Firmicutes & 0.745517928 \\
\hline S20 & Male & $42 y$ & H (1 day) & Kolkata & VCO1 Ogawa & Firmicutes & 0.043091111 \\
\hline
\end{tabular}




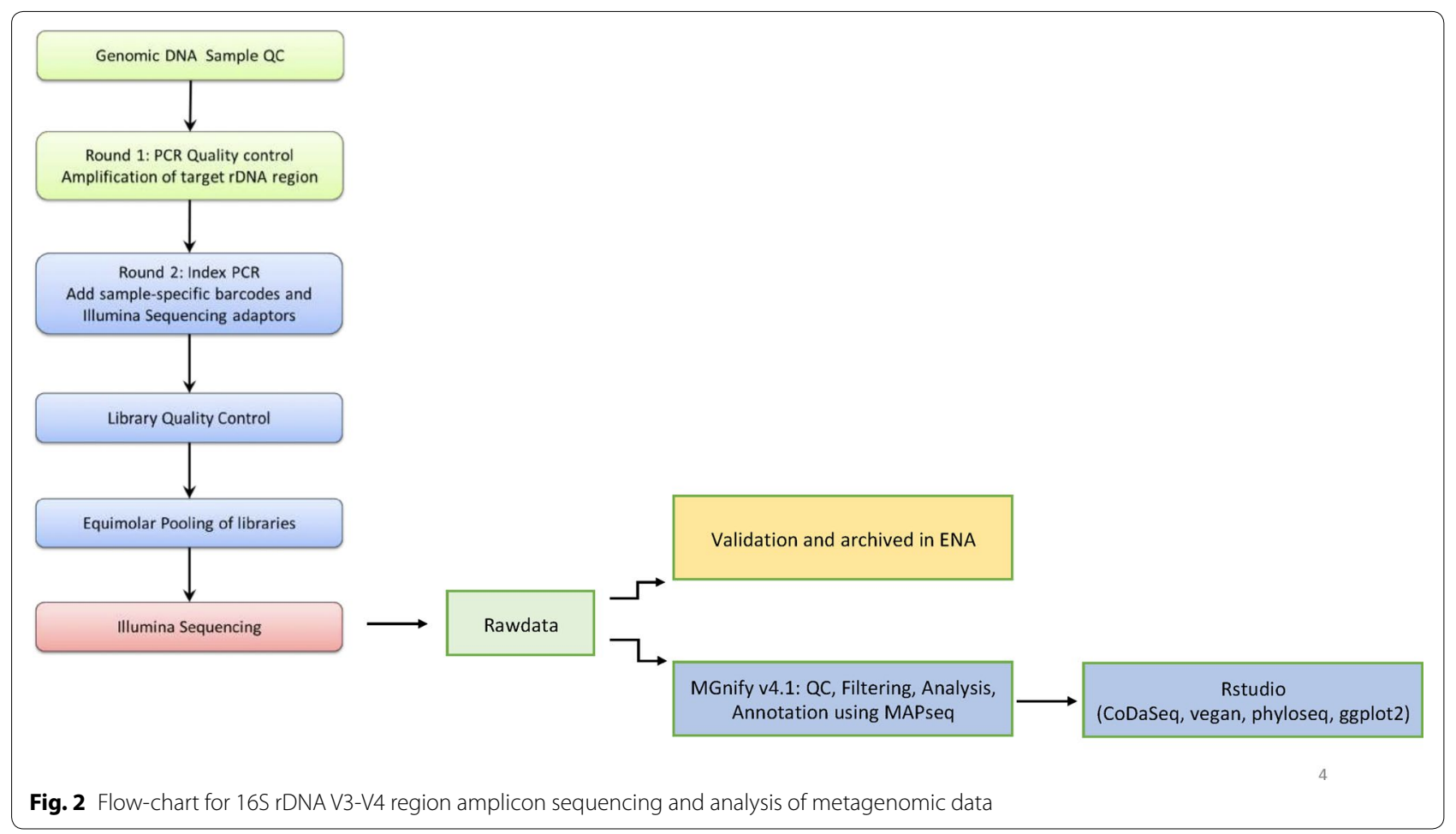

of Bacteroidetes and 20.71\% of Firmicutes. Bacteroidetes was the most dominant phylum only in S13 (28.87\%). S13 was associated with VC O139. Table 1 shows the most abundant phylum present in each sample S1-S20. Figure 4 shows the relative abundance (in percentage) of the major phyla in each sample from S1 to S20. A large proportion of reads in every sample could not be assigned any taxonomic rank below domain and was labeled as unassigned bacteria (Fig. 4). The mean of abundance of various phyla in 20 samples was 38\% Firmicutes, 10\% Bacteroidetes, 12\% Actinobacteria and 19\% Proteobacteria. Verrucomicrobia, Fusobacteria, Tenericutes, Spirochaetes, Lentisphaerae, Elusimicrobiae, Cyanobacteria, Synergistetes, Deferribacteres, Acidobacteria, Armatimonadetes, Caldotrichaeota, Chloroflexi, Deinococcus, Fibrobacteres, Gemmatomonadetes, Ignavibacteriae, Nitrospinae, Kiritimatiellaeota, Planctomycetes, Candidate Phyla Radiation (CPR) also appeared in many samples. Candidatus Saccharibacteria or TM7 phylum, was detected in many samples like S2 (0.04\%), S3 (0.03\%), S7 (0.01\%), S8 (0.03\%), S11 (0.01\%), S13 (0.01\%), S14 (0.02\%). Verrucomicrobiae formed $19.91 \%$ of $S 5,13.4 \%$ of $S 13$ and $11.94 \%$ of S20. From all the three samples VC was isolated as the diarrheal agent. In S1 and S2 which were also associated with VC Verrucomicrobia was present at an abundance of $>0 \%-<1 \%$. Table 2 presents a catalogue of the different phyla found in the study cohort.
Different bacterial classes were found in variable proportion in the 20 samples. Actinobacteria, Bacilli, Bacteroidia, Coriobacteria, Clostridia, $\gamma$-Proteobacteria and Verrucomicrobiae were the most prominent classes observed. Bacilli was the most dominant class in seven samples (S3, S7,S8, S10, S11, S14, S16). S1 was mainly composed of unclassified bacteria and $\gamma$-Proteobacteria is the only annotated class that is present in high proportion but $<50 \%$. In this sample all other classes are present in lower proportion. In S18 $\gamma$-Proteobacteria was found in relative abundance of $>50 \%$. Other samples where $\gamma$-Proteobacteria was present prominently but at $<50 \%$ relative abundance were S2, S3, S4, S6, S8, S10, S11, S12, S13, S14, S16, S17, S19, S20.

S7, S9, S17 showed the presence of 25\% Erysipelotricha while S2, S10, S15 and S19 have $<25 \%$. Classes that were found in $>0 \%-<1 \%$ abundance in many of the samples were Acidimicrobia, Rubrobacteria, Armatimonadia, Cytophagia, Flavobacteria, Calditrichae, Anaerolineae, Deinococci, Negativicutes, Tissierellia, Fusobacteria, $\alpha$, $\beta, \delta, \varepsilon, \zeta$ - Proteobacteria, Fimbrimonadia, Nitriloruptoria, Ktedonobacteria, Sphingobacteria, Fibrobacteria, Gemmatimonadetes, Ignavibacteria, Lentisphaeria, Phycisphaerae, Opitutae, Endomicrobia, Spiritrichae, Saprospiria, Oligoflexia, Oligosphaeria, Spirochaetia, Synergistia, Mollicutes, Chloroflexia, Elusimicrobia, Acidithiobacillia, Solibacteres, Chitiniphagia, Chlamydiia, 


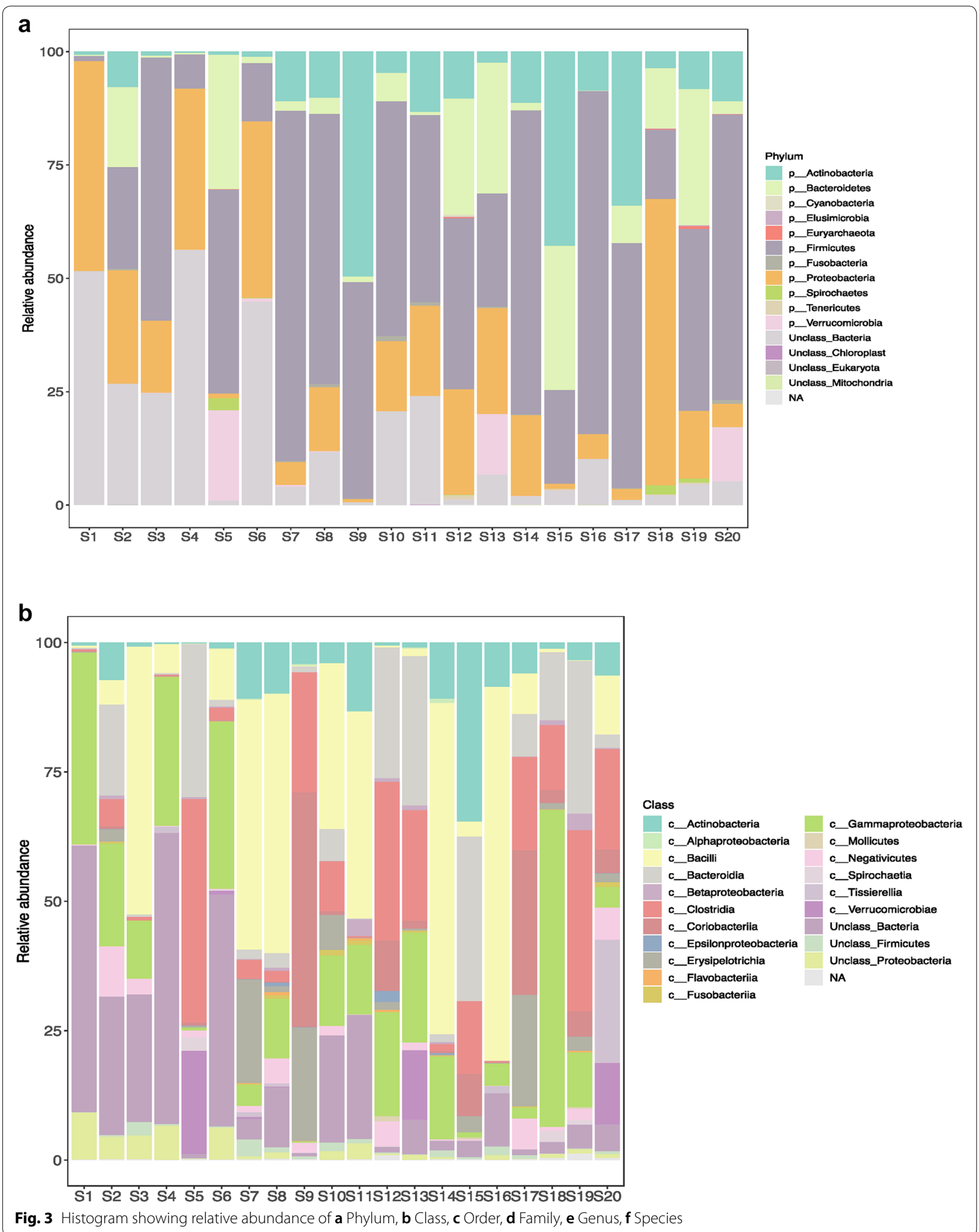




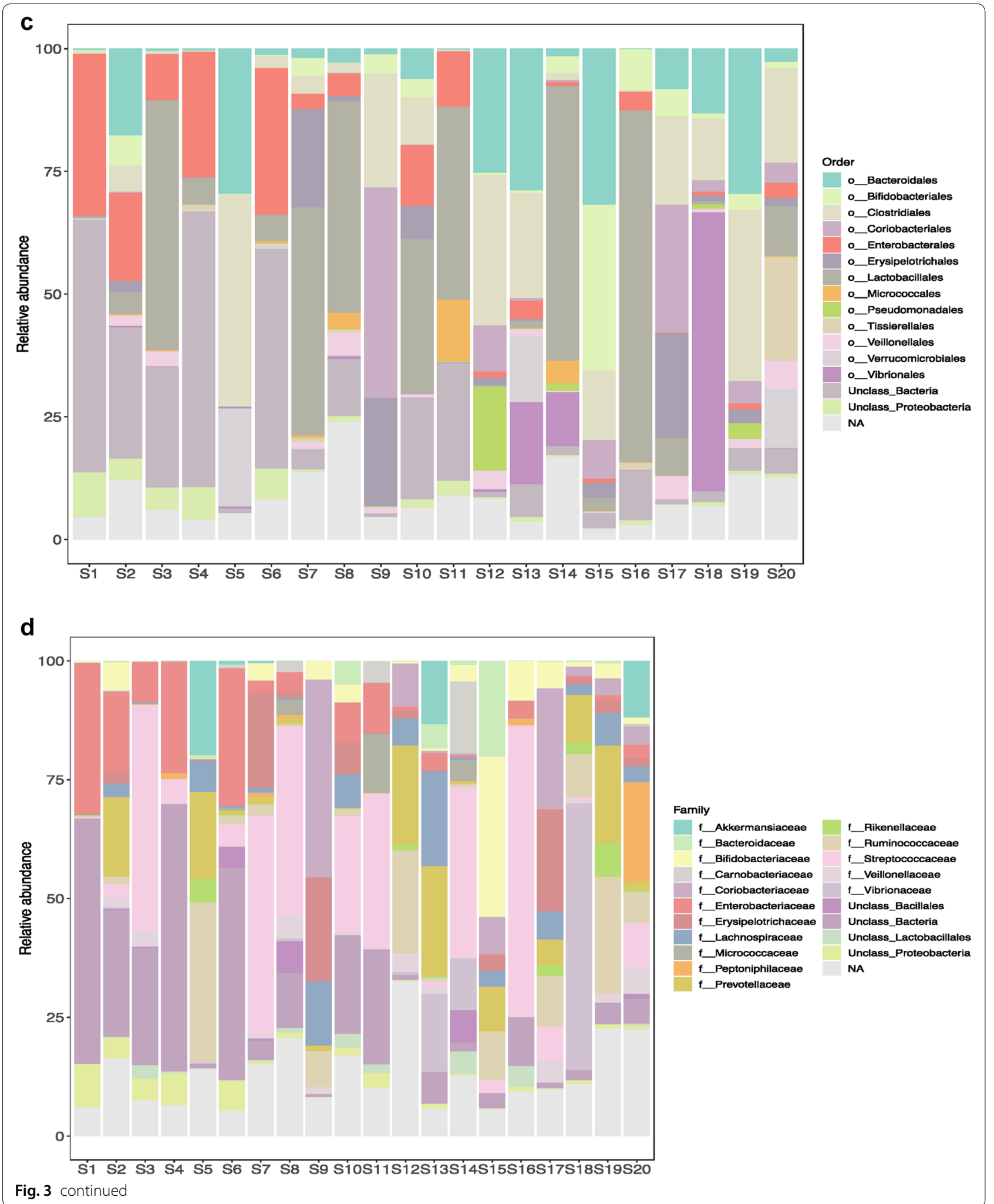




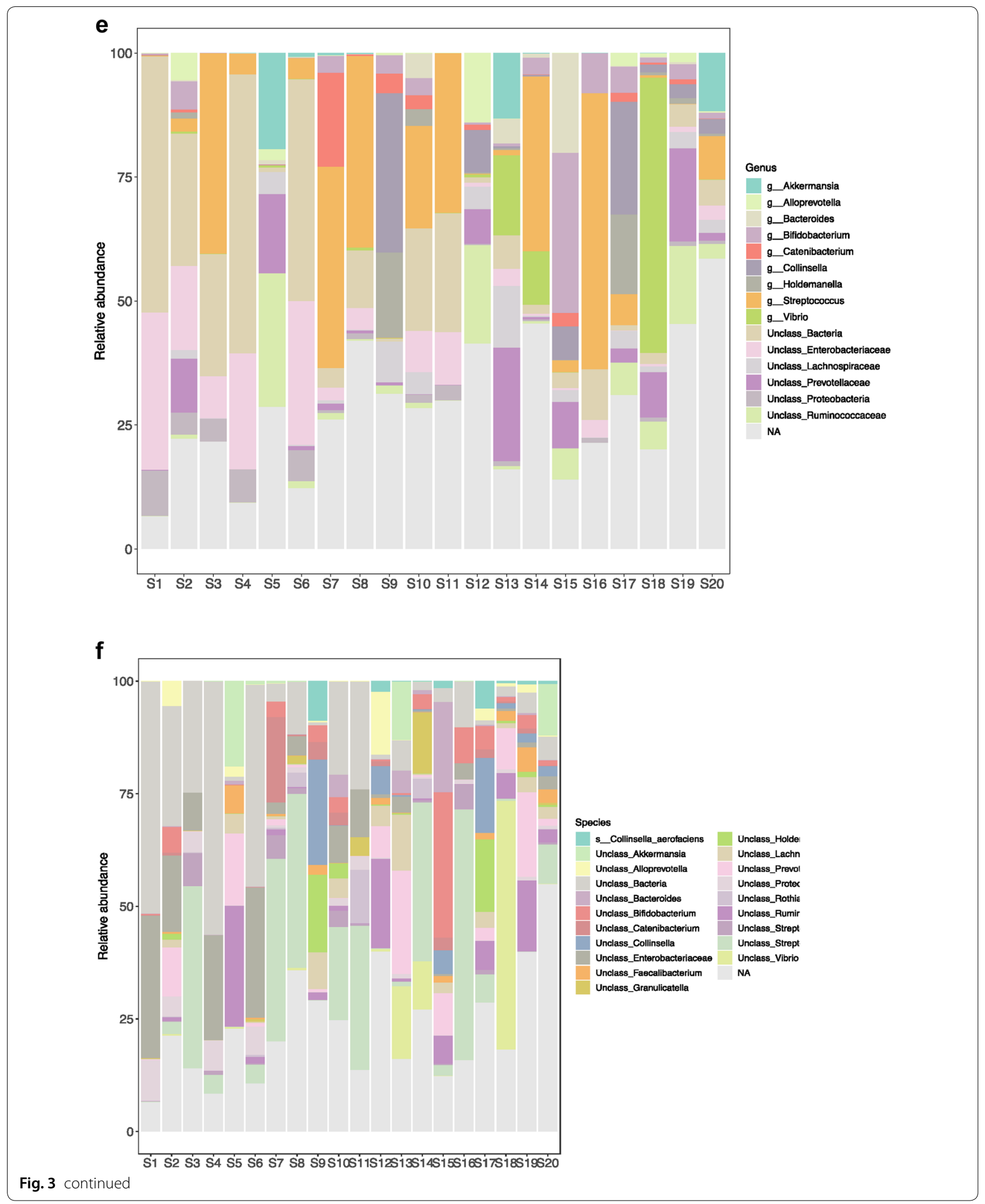

Genus 


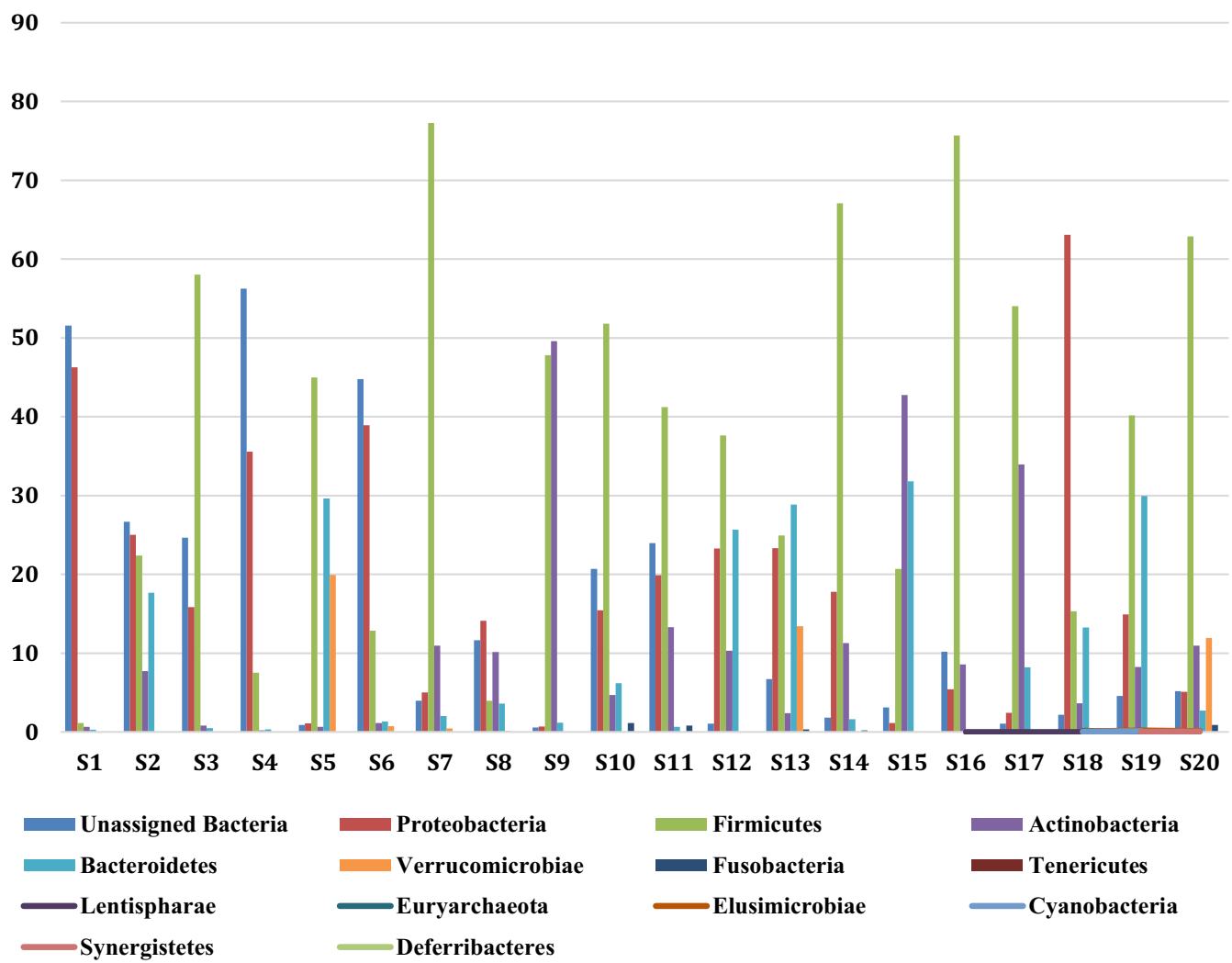

Fig. 4 Relative abundance of the major bacterial phyla in the diarrheal gut microbiome. Bar-diagram showing relative abundance of the major bacterial phyla in each diarrheal sample

Kiritimatiella, Halobacteria, Caldilineae, Dehalococcoidea, Thermomicrobia, Limnochordia, Planctomycetia, Hydrogenophilalia, Balneolia, Spartobacteria, Holophagae, Thermideophilia, Longimicrobia.

Table 2 presents a list of all the different orders reported from this study. Order Actinomycetales, Bacteroidales, Enterobacterales, Bifidobacteriales, Corynebacteriales, Micrococcales, Clostridiales, Coribacteriales, Erysipelotrichales, Lactobacillales, Pseudomonadales, Tissierellales, Verrucomicrobiales, Vibrionales, Streptomycetales, Flavobacteriales, Bacillales, Selenomonadales, Fusobacteriales, Rhizobiales, Rhodobacterales, Burkholderiales, Neisseriales, Desulfovibrionales, Myxococcales, Campylobacterales, Aeromonadales, Cellvibrionales, Chromatiales, Pasteurellales, were found in variable proportion in all the twenty samples. In S7, S8, S14 and S20 Actinomycetales was found at $2 \%-6 \%$ abundance. In S5, S13, S15, S19 Bacteroidales were observed at $>25 \%$ abundance. Abundance of Enterobacterales in S5, S9, S17, S18, $\mathrm{S} 19$ was $>0 \%-1 \%$. In all others it was between 1 and $25 \%$. Bifidobacteriales was present at $>5 \%$ abundance in $\mathrm{S} 2$, S7, S9, S10, S14, S15, S16, S17, S18, S19, S20 with $>25 \%$ abundance in S15. In all other samples its abundance was between $0 \%$ and $1 \%$. The abundance of Micrococcales was as high as 3.3\% in S8, 12.5\% in S11 and 4.6\% in S14. Clostridiales was a dominant order in S5, S9, S10, S12, S13, S15, S17, S18, S19, S20 where its abundance rate was $8 \%-40 \%$ while in S2, S6, S7, S8, S14 they were present at $1 \%-2 \%$ abundance. Abundance of $3 \%-44 \%$ Coriobacteriales was observed in S9, S12, S15, S17, S18, S19, S20 while in all other samples its abundance rate was $<1 \%$. Proportion ranging from $1 \%$ to $21.5 \%$ of Erysipelotrichales was present in S2, S7, S8, S9, S10, S12, S15, S17, S18, S19 and S20. In the remaining samples it occurred at $<1 \%$ abundance. A proportion of the diarrheal microbiota comprised Lactobacillales in a majority of the samples. These were S3 (>50\%), S4 (>5.5\%), S6 (>5.3\%), S7 (>46.5\%), S8 (>42\%), S10 (31.4\%), S11 (>39\%), S13 (>1.4\%), S14 (>55.7\%), S15 (>2.8\%), S16 (>71.7\%), S17 (>7.6\%), S20 $(>10.1 \%)$. In the remaining samples presence of Lactobacillales was found at an abundance rate of $<1 \%$ Pseudomonadales was conspicuous in S12 (17.1\%), S14 (1.5\%), S19 (3.2\%) and Tissierellales in S20 (21.1\%). In S5, S13 and S20 order Verrucomicrobiales was present at 19.9\%, $13.4 \%$ and $11.9 \%$ respectively. Vibrionales were conspicuously abundant in S13, S14 and S18 and were found 
Table 2 Catalogue of phyla, orders, families found in the study cohort

\section{Phylum present in all subjects}

Firmicutes, Bacteroidetes, Actinobacteria, Proteobacteria

\section{Phylum not present in all subjects}

Verrucomicrobia, Fusobacteria, Tenericutes, Spirochaetes, Lentisphaerae, Elusimicrobiae, Cyanobacteria, Synergistetes, Deferribacteres, Acidobacteria, Armatimonadetes, Caldotrichaeota, Chloroflexi, Deinococcus-Thermus, Candidatus, Fibrobacteres, Gemmatomonadetes, Ignavibacteriae, Nitrospinae, Kiritimatiellaeota, Planctomycetes, Balneolaeota, Chlamydiae,

Candidatus Falkowbacteria, Candidatus Moranbacteria, Candidatus Saccharibacteria, Candidatus Latescibacteria,

Candidatus Melainabacteria,Candidatus Peregrinibacteria, Thermodesulfobacteria, Candidatus Shapirobacteria, Candidatus Jorgensenbacteria, Candidatus Raymondbacteria, Candidatus Schekmanbacteria, Candidatus Doudnabacteria, Candidatus Gracilibacteria, Candidatus Portnoybacteria, Candidatus Yanofskybacteria,Candidatus Parcubacteria, Candidatus Wolfebacteria,Candidatus Lindowbacteria,

Candidatus Pacebacteria
Orders present in all subjects

Actinomycetales, Bacteroidales, Enterobacterales, Bifidobacteriales, Corynebacteriales, Micrococcales, Clostridiales, Coribacteriales, Erysipelotrichales, Lactobacillales, Pseudomonadales, Tissierellales, Verrucomicrobiales, Vibrionales, Streptomycetales, Flavobacteriales, Bacillales, Selenomonadales, Fusobacteriales, Rhizobiales, Rhodobacterales, Burkholderiales, Neisseriales, Desulfovibrionales, Myxococcales, Campylobacterales, Aeromonadales, Cellvibrionales, Chromatiales, Pasteurellales

\section{Orders not present in all subjects}

Veillonellales, Propionibacteriales, Eggerthellales, Pseudonocardiales, Gaiellales, Armatimonadales, Oceanospirillales, Cytophagales, Acidaminococcales Rhodospirillales, Rickettsiales, Sphingomonadales, Xanthomonadales, Oligoflexales, Sphaerobacterales, Kallotenuales, Chroococcales, Calditrichales, Anaerolineales, Deinococcales, Caulobacterales, Nitrosomonadales, Bradymonadales, Desulfobacterales, Alteromonadales, Arenicellales, Cardiobacteriales, Legionellales, Immundisolibacterales, Thiotrichales, Mariprofundales, Spirochaetales, Synergistales, Mycoplasmatales, Acidimicrobiales, Acidothermales, Frankiales, Kineosporiales, Euzybyales, Gaiellales, Marinilabiliales, Deferribacterales, Fibrobacterales, Gemmatimonadales, Victivallales, Acidithiobacillales, Holosporales, Rhodocyclales, Desulfuromonadales, Acholeplasmatales, Methylococcales, Bacteriovoracales, Bdellovibrionales, Brachyspirales, Anaeroplasmatales, Opitutales, Puniceicoccales, Saprospirales, Halanaerobiales, Ignavibacteriales, Planctomycetales, Magnetococcales, Haloplasmatales, Acidobacterales, Solibacterales, Rubrobacterales, Chitinophagales, Sneathellales, Micromonosporales, Parachlamydiales, Caldilineales, Thermomicrobiales, Limnochordales, Chthoniobacterales, Jiangellales, Streptosporangialles, Thermoanaerobacterales, Kordiimonadales, Acidiferrobacterales, Nakamurellales, Solirubrobacterales, Dehalococcoidales, Endomicrobiales, Holophagales, Ardenticatenales, Nostocales, Gloebacterales, Parvularculales, Pelagibacteriales, Natranaerobiales, Desulfarculales, Syntrophobacterales, Synechococcales, Geodermatophilales, Methylacidophilales, Kiloniellales, Hydrogenophilales, Nitriliruptorales, Balneolales, Ktedonobacterales, Salinisphaerales, Chloroflexales, Ferrovales, Orbales, Nitriruptorales, Fimbrimonadales, Oligosphaerales, Nitrospirales, Neviskiales, Entomoplasmatales, Acanthopleuribacterales, Pleurocapsales Longimicrobiales. 
Table 2 (continued)

\section{Families present in all subjects}

Actinomycetaceae, Bifidobacteriaceae, Corynebacteriaceae, Microbacteriaceae, Micrococcaceae, Streptomycetaceae, Atopobiaceae, Coriobacteriaceae, Bacteroidaceae, Prevotellaceae, Rickenellaceae, Flavobacteriaceae, Bacillaceae, Staphylococcaceae, Aerococcaceae, Carnobacteriaceae, Enterococcaceae, Lactobacillaceae, Streptococcaceae, Christencenellaceae, Clostridiaceae, Clostridiales Family XIII Incertae Sedis, Lachnospiraceae, Peptococcaceae, Peptostreptococcaceae, Ruminococcaceae, Erysipelotrichaceae, Selenomonadaceae, Veillonellaceae, Peptoniphilaceae, Fusobacteriaceae, Methylobacteriaceae, Rhodobacteraceae, Neisseriaceae, Campylobacteraceae, Succinivibrionaceae, Enterobacteriaceae, Pasteurellaceae, Vibrionaceae, Akkermansiaceae

\section{Families not present in all subjects}

Solibacteraceae, Gordoniaceae, Dietziaceae, Nocardiaceae, Brevibacteriaceae, Dermacoccaceae, Dermatophilaceae, Intrasporangiaceae, Nocardioidaceae, Propionibacteriaceae, Pseudonocardiaceae, Eggerthellaceae, Dysgomonadaceae, Lentimicrobiaceae, Muribaculaceae, Odoribacteraceae, Paludibacteraceae, Prolixibacteraceae, Porphyromonadaceae, Tannerellaceae, Cytophagaceae, Microscillaceae, Calditrichaceae, anaerolineaceae, Deinococcaceae, Saprospiraceae, Alicyclobacilaceae, Planococcaceae, Leuconostocaceae, Caldicoprobacteraceae, Eubacteriaceae, Oscillosporaceae, Syntrophomonadaceae, Acidaminococcaceae, Leptotrichiaceae, Caulobacteraceae, Hyphomonadaceae, Erythrobacteraceae, Sphingomonadaceae, Burkholderiaceae, Comamonadaceae, Suttarellaceae, Nitrosomonadaceae, Bradymonadaceae, Desulfobulbaceae, Desulfovibrionaceae, Helicobacteraceae, aeromonadaceae, Shewanellaceae, Arenicellaceae, Cardiobacteriaceae, Helieaceae, Spongibacteraceae, Chromatiaceae, Erwiniaceae, Morganellaceae, Pectobacteriaceae, Yersiniaceae, Immundisolibacteraceae, Coxiellaceae, Alcanivoraceae, Endozoicomonaceae, Halomonadaceae, Oceanospirillaceae, Thiotrichaceae, Xanthomonadaceae, Oligoflexaceae, Mariprofundaceae, Spirochaetaceae, Synergiataceae, Mycoplasmataceae, Fabaceae, Acidimicrobiaceae, Mycobacteriaceae, Sphingobacteriaceae, Sporichthyaceae, Kineosporiaceae, Bogoriellaceae, Dermabacteraceae, Microcystaceae, Deferribacteraceae, Elusimicrobiaceae, Fibrobacteraceae, Defluvitaleaceae, Thermoactinomycetaceae, Sporomusaceae, Gemmatimonadaceae, Acidithiobacillaceae, Candidatus Paracaedibacteraceae, Hyphomicrobiaceae, Acetobacteraceae, Geminicoccaceae, Rickettsiaceae, Oxalobacteraceae, Thiobacillaceae, Azonexaceae, Rhodocyclaceae, Zoogloeaceae, Desulfomicrobiaceae, Desulfuromonadaceae, Geobacteraceae, Alteromonadaceae, Moritellaceae, Porticoccaceae, Halothiobacillaceae, Hafniaceae, Legionellaceae, Methylococcaceae, Bactriovoracaceae, Bdellovibrionaceae, Brachyspiraceae, Anaeroplasmataceae, Opitutaceae, Puniceicoccaceae, Frankiaceae, Promicromonosporaceae, Listeriaceae, Paenibacillaceae, Sporolactobacillaceae, Halanaerobiaceae, Halobacteroidaceae, Ignavibacteriaceae, Magnetococcaceae, Chromobacteriaceae, Desulfobacteriaceae, Sandaracinaceae, Williamsiaceae, Gemmataceae, Rhodospirillaceae, Kofleriaceae, Pseudoalteromonadaceae, Microbulbiferaceae, Hanellaceae, Saccharospirillaceae, Rhodoanobacteraceae, Bryobacteraceae, Beijerinckiaceae, Sneathiellaceae, Haloplasmataceae,Tsu kamurellaceae, Micromonosporaceae, Barnesiellaceae, Cyclobacteraceae, Parachlamydiaceae, Caldiliniaceae, Aurantimonadaceae, Rhizobiaceae, Ectothiorhodospiraceae, Jiangellaceae, Cellumonadaceae, Rubrobacteraceae, Chitinophagaceae,

Bradyrhizobiaceae, Brucellaceae, Cellvibrionaceae, Nakamurellaceae, Segniliparaceae, Amoebophilaceae, Cryomorphaceae, Endomicrobiaceae, Pasteuriaceae, Clostridiales Family XVIII Incertae Sedis, Gracilibacteraceae, Thermodesulfobiaceae, Isophaeraceae, Planctomycetaceae, Anaplasmataceae, Candidatus Midichloriaceae, Methylophilaceae, Polyangiaceae, Oleiphilaceae, Leptospiraceae, Verrucomicrobiaceae, Holophagaceae, Crocinitomicaceae, Gottschalkiaceae, Victivallaceae, Parvularculaceae, Alcaligenaceae, Competibacteraceae, Psychromonadaceae, Woeseiaceae, Acholeplasmataceae, Sanguibacteraceae, Thermoanaerobacterales Family III Incertae Sedis, Desulfarculaceae, Geodermatophilaceae, Natramaerobiaceae, Liminochordaceae,Anaeromyxobacteraceae, Hymenobacteraceae, Trueperaceae, Archangiaceae, Rubritaleaceae, Idiomarinaceae, Hydrogenophilaceae, Nitriliruptoraceae, Flammeovirgaceae, Ichthyobacteriaceae, Proteinivoraceae, Rhodobiaceae, Xanthobacteraceae, Chlamydiaceae, Orbaceae, Blattabacteriaceae, Nitrospiraceae, Chthoniobacteraceae, Cohaesibacteraceae, Acanthopleuribacteraceae, Parviterribacteraceae, Xenococcaceae, Longimicrobiaceae, Galiionellaceae, Syntrophaceae, Thorselliaceae, Beutenbergiaceae, Thermoanaerobacterales, Family IV Incertae Sedis, Phyllobacteriaceae, Sterolibacteriaceae, Ferrimonadaceae. at $16.7 \%, 11 \%$ and $56.9 \%$ respectively. Bacillales were present above 1\% in S6 (4.3\%), S8 (6.7\%), S14 (7.78\%).
Selenomonadales was present at $6.2 \%$ in S2. Burkholderiales and Neisseriales were present at $1 \%$ and $1.9 \%$ 
respectively in S19. 2\% Campylobacterales was present in S12. Aeromonadales were present at $1.3 \%$ in S6 and 5.5\% in S19. Pasteurellales were present at $6 \%$ in S8, $1.5 \%$ in S14 and $1.8 \%$ in S17.

Veillonellales were completely absent in S1. It was found in the remaining samples. Its proportion in some of the samples were as follows: S2 (2\%), S3 (3\%), S7 (1.2\%), S8 (4.8\%), S9 (1.2\%), S10 (0.7\%), S12 (3.7\%), S13 (1.4\%), S14 (0.25\%), S17 (4.5\%), S18 (0.63\%), S19 (1.8\%), S20 (5.7\%). Propionibacteriales and Eggerthellales were absent in S4, Pseudonocardiales were absent in S3, S5, S9, S10, S12, S18, S19, Gaiellales were absent in S1, S3, S4, S8, S14 and S20. Armatimonadales were absent in S1, S2, S3, S4, S7, S8, S12, S18, S19. Oceanospirillales were absent in S16. Cytophagales were found to be absent in S4, S11 and S18. Acidaminococcales and Rhodospirillales were absent in S3. Rickettsiales were absent in S1 and S7. Sphingomonadales was absent in S5. Xanthomonadales and Oligoflexales were absent in S5 and S9. Sphaerobacterales were present only in S10 and Kallotenuales in S18 only. Chroococcales were found in S14, S17 and S18. Other orders that appear in Table 2 were observed in some samples in minute proportion.

Table 2 shows the different bacterial families that were found in the study. Streptococcaceae was the dominant family in $35 \%$ of samples, in $10 \%$ samples Coriobacteriaceae was dominant and 5\% samples Vibrionaceae was dominant. S1 consisted of predominantly Enterobacteriaceae and Unclassified Bacteria. Actinomycetaceae, Bifdobacteriaceae, Corynebacteriaceae, Coriobacteriaceae, Bacteroidaceae, Prevotellaceae, Streptococcaceae, Ruminococcaceae, Erysipelotrichaceae, Veillonellaceae, Enterobacteriaceae, Pasteurellaceae, Vibrionaceae, Lachnospiraceae were found at greater than $1 \%$ in many samples (Fig. 5). In all other samples all these families occurred at a relative abundance of less than $1 \%$.

Table 3 presents the genera and species under Kingdom Bacteria that were found in the study cohort. A total of 584 genera were observed. 136 of these could be further classified till the species level while the remaining 448 could not be classified further with $16 \mathrm{~S}$ rDNA amplicon sequencing. Akkermansia sp., Alloprevotella sp., Bacteroides sp., Bifidobacterium sp., Catenibacterium sp., Collinsella sp., Holdmanella sp., Streptococcus sp., Vibrio sp. occurred at $1 \%$ and greater relative abundance. Genera present in all the 20 diarrheal samples were Actinomyces sp., Bifidobacterium sp, Corynebacterium sp., Bacteroides sp., Alloprevotella sp., Lactobacillus sp., Streptococcus sp., Clostridium sp., Blautia sp., Peptostreptococcus sp., Faecalibacterium sp., Holdemanella sp., Dialister sp., Methylobacterium sp., Neisseria sp., Acinetobacter sp., Vibrio sp., Akkermansia sp. Akkermansia sp. was found at $<1 \%$ in all the samples except S5, S13 and S20. The relative abundance of Akkermansia sp. in S5 was 19.2\%, in S13 was $13.1 \%$ and in S20 was $11.6 \%$. Clostridium sp. was found at $<1 \%$ abundance in all the 20 samples. Bifidobacter sp. was present at 32\% in S15. In all other samples its abundance was below 10\%. A complete hierarchical classification of the different microbial units found in the study by $16 \mathrm{~S}$ rDNA sequence homology has been presented in Additional file 1.

\section{Correlation of commensal and pathogen abundance in diarrhea}

Differences in relative abundance of four different families namely Bifidobacteriacea, Enterobacteriaceae, Bacteroidaceae and Vibrionaceae in diarrheal samples S1 to S20 were observed and graphically presented in Fig. 6A(a). Families Bifidobacteriaceae and Enterobacteriaceae were negatively correlated with $r_{s}-0.40695$ and 2 -tailed $p$ value of 0.07495 . The association was non-significant. Negative correlation between Bifidobacteriaceae and Vibrionaceae was found at $r_{s}-0.03073$ and the association was non-significant with 2 -tailed $p$ value of 0.8977, Streptococcaceae and Enterobacteriaceae were found to be positively correlated with $r_{s}=0.29959$ and the association was found to be non-significant with a 2-tailed $p$ value of 0.19941 .

A significant positive correlation with $r_{s} 0.4751$ and a two-tailed p-value of 0.0343 between Bifidobacteriaceae and Lachnospiraceae, significant negative correlation with $r_{s}-0.6338$ and -0.6882 and two-tailed p-values of 0.0027 and 0.0008 respectively between Enterobacteriaceae and Lachnospiraceae and Enterobacteriaceae and Ruminococcaceae, significant positive correlation between Lachnospiraceae and Ruminococcaceae with $r_{s} 0.7111$ and two-tailed p-value of 0.0004 and significant negative correlation between Lachnospiraceae and Streptococcaceae with $r_{s}-0.5215$ and two-tailed p-value of 0.0184, significant negative correlation was found between Ruminococcaceae and Streptococcaceae with $r_{s}$ -0.6847 and two-tailed p-value of 0.0009. The Spearman's rank correlation coefficient and two-tailed p-values have been represented graphically in Fig. $6 \mathrm{a}$.

\section{Difference in abundance of commensals and pathogens in diarrhea}

Kruskal-Wallis test performed to compare difference in abundance among families of commensals namely, Bifidobacteriaceae, Ruminococcaceae and Lachnospiraceae in diarrhea showed a positive trend with $\mathrm{H}$ statistic 1.5543 $(2, N=60)$ and with a p-value of 0.4597 . Kruskal-Wallis test performed to compare differences among families of pathogens namely, Bacteroidaceae, Enterobacteriaceae 


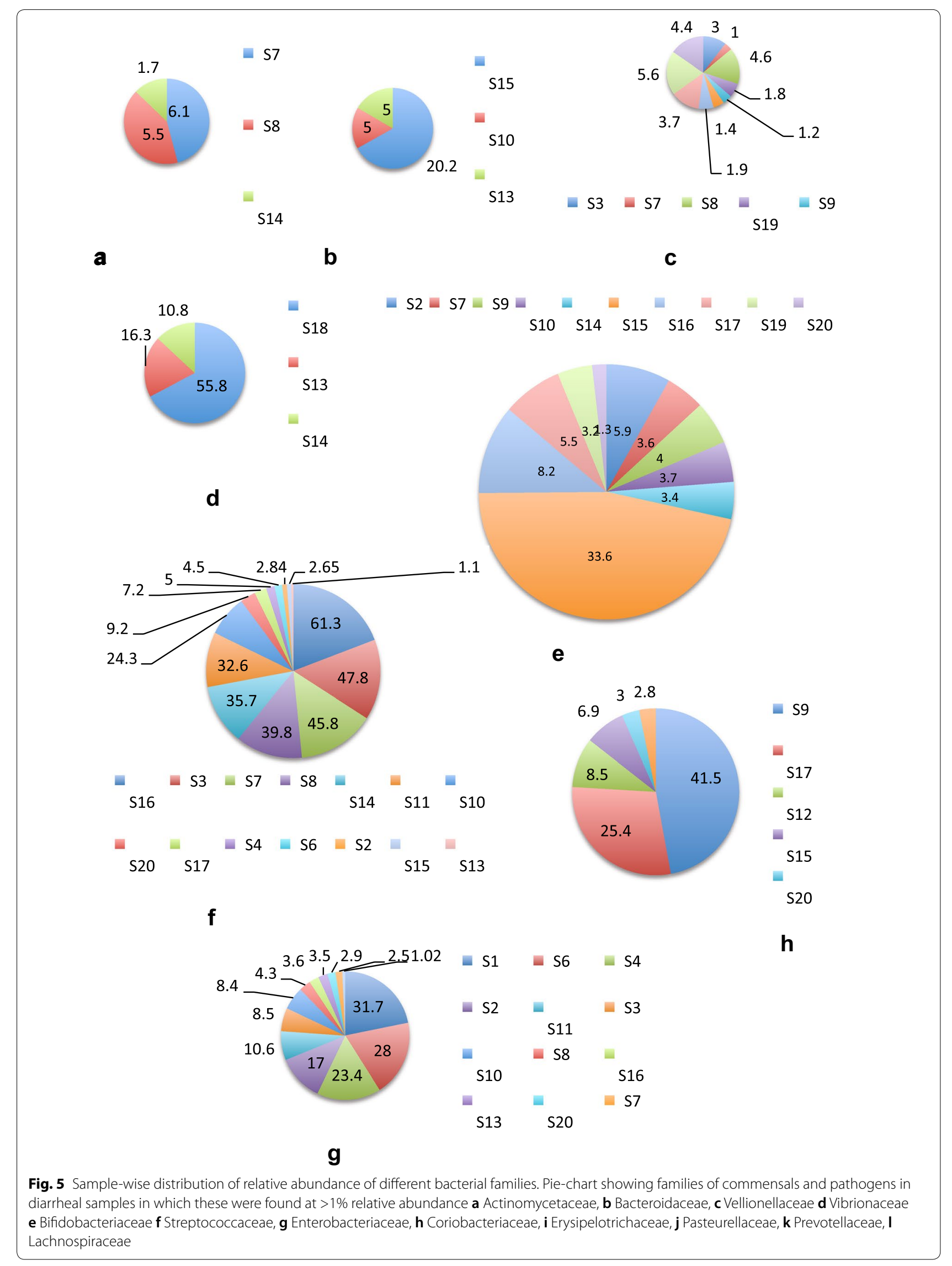



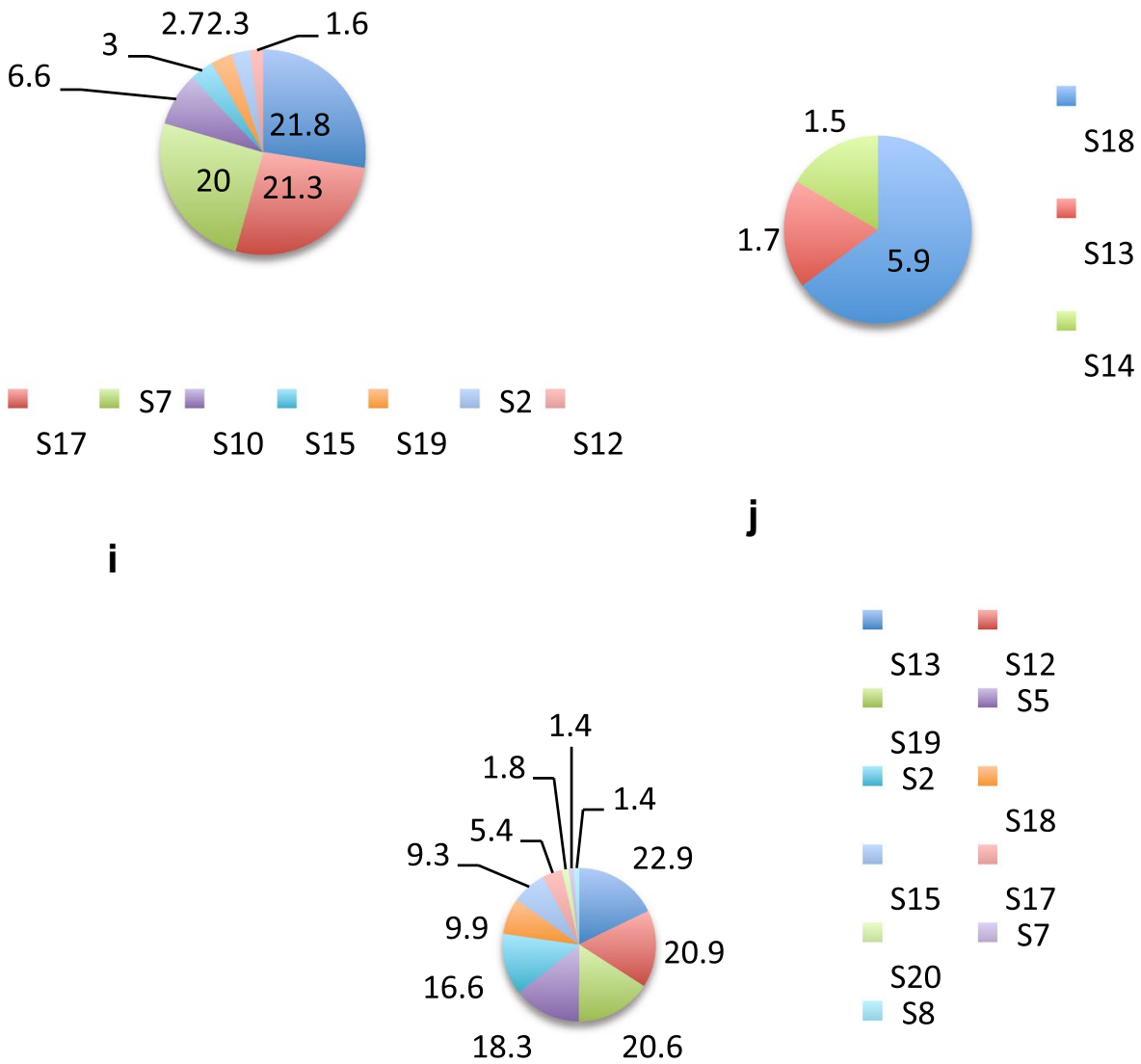

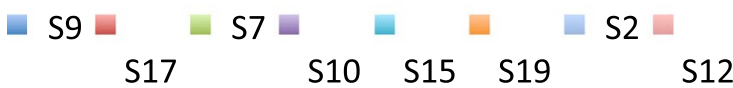

i

k
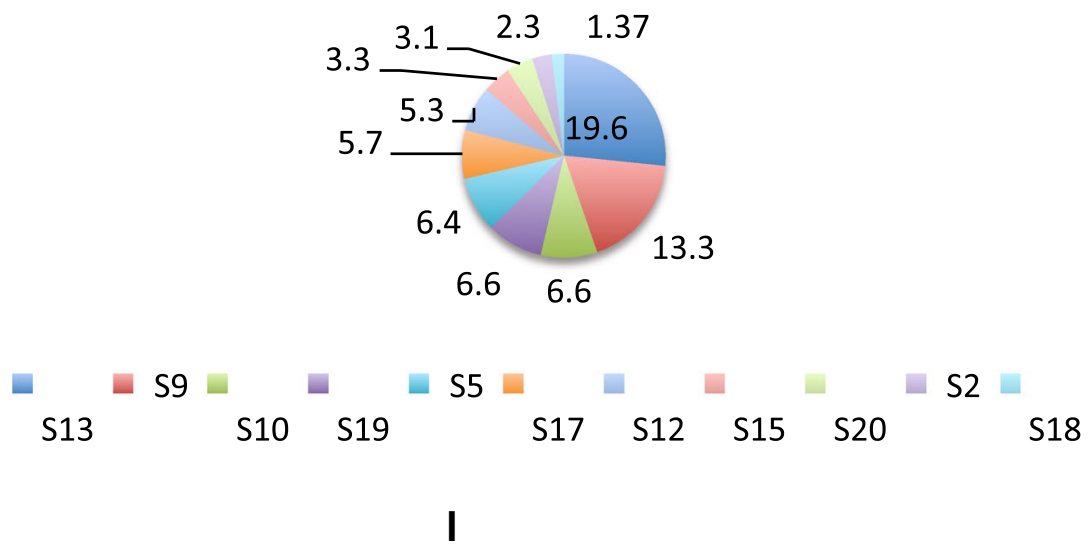

Fig. 5 continued

and Vibrionaceae showed a significant difference with $\mathrm{H}$ statistic $21.574(2, N=60)$ with p-value of 0.00002 .

Unpaired t-test with Wilcoxon matched-pairs signed rank test was used to calculate and compare the difference of relative abundance of family Bifidobacteriaceae and Enterobacteriaceae, Bifidobacteriaceae and Vibrionaceae, Lachnospiraceae and Vibrionaceae, Lachnospiraceae and Enterobacteriaceae, Enterobacteriaceae and Vibrionaceae and Aeromonadaceae and Enterobacteriaceae. Figure 6B shows the differences in 
Table 3 Genus and species catalogue

\section{Genus}

Blastocatella sp.

Actinomyces sp.*

canobacterium sp.

Bifidobacterium sp.*

$$
\begin{aligned}
& \text { B. longum } \\
& \text { B. ramosum } \\
& \text { B. secularae } \\
& \text { B. bifidum } \\
& \text { B. pullorum } \\
& \text { B. sp. MRM } 8.19 \\
& \text { B. aerophilum } \\
& \text { B. angulatum } \\
& \text { B. animalis } \\
& \text { B. avesanii } \\
& \text { B. biavatii } \\
& \text { B. sp. MC10 }
\end{aligned}
$$

Corynebacterium sp.*

$$
\begin{aligned}
& \text { C. falsenii } \\
& \text { C. bovis } \\
& \text { C. accolens } \\
& \text { C. kroppenstedtii } \\
& \text { C. cystitidis } \\
& \text { C. sp. M72 } \\
& \text { C. doosarense } \\
& \text { C. simulans } \\
& \text { C. sp. NML98-0116 } \\
& \text { C. glaucum } \\
& \text { C. sp. } 1938 B R R J \\
& \text { C. sp321002 } \\
& \text { C. sp. NML080024 } \\
& \text { C. durum }
\end{aligned}
$$

Turicells sp.

$$
\text { T. otidis }
$$

Dietzia sp.

Rhodococcus sp.

Brevibacterium sp.

$$
\text { B. Iuteolum }
$$

Kytococcus sp.

Candidatus Planktoluna sp.

Microbacterium sp.

Micrococcus sp.

Rothia sp

\section{Samples carrying these species}

\author{
$\mathrm{S} 13, \mathrm{S7}, \mathrm{S} 8$ \\ $\mathrm{S} 7, \mathrm{~S} 8, \mathrm{~S} 14$
}

58

S16, S9, S17, S15, S10

$\mathrm{S} 16, \mathrm{~S} 15$

S7

$\mathrm{S} 17$

S17

S2

$\mathrm{S} 15$

S2, S9, S10, S15, S19, S17

$\mathrm{S} 15, \mathrm{~S} 10$

S15

S15

S15, S17

53

S16

S8, S10, S13, S14

S9, S20, S10, S13

S20

$\mathrm{S} 10$

S1

$\mathrm{S} 14$

S16

S7

S7, S11, S13, S14

$\mathrm{S7}, \mathrm{S} 8$

S7

S20

S1, S3, S4, S7, S14, S15, S20
M. terreus

R. dentocariosa

R. aeria

R. sp. HMSC065C03 
Table 3 (continued)

\section{Genus}

Friedmanniella sp.

Mamoricola sp.

Cutibacterium sp.

Streptomyces sp.

Libanicoccus sp.

Enterorhabdus sp.

slckia sp.

Bacteroides sp. *

Butyricimonas sp.

Odoribacter sp.

Porphyromonas sp.

P. sp. oral taxon $278 \mathrm{~K}$

Alloprevotella sp.*

\section{A. tannerae}

A. rava

Prevotella sp.

Parabacteroides sp.

Flectobacillus sp.

Bergeyella sp.

P. enoeca
P. micans
P. sp. AN5135
P. copri
P. sp. oral clone ASCG10
P. sp. HJM029
P. sp. $310-5$
P. marshii
P. nigrescens
P. stercorea
P. sp. 152 R-1a
P. intermedia
P. sp. HUN102
P. sp. oral cloneKW035

B. plebius

B. fragilis

B. propionicifaciens

B. sp. CAG:1060_57_27

B. vulgatus

B. acidifaciens

B. paurosaccharolyticus

$\mathrm{S} 13, \mathrm{~S} 14$

S18, S19, S9, S17, S12, S13

S15, S17, S20, S12, S10, S13

S7, S10,

S15, S19

$\mathrm{S} 10, \mathrm{~S} 15$

S5

$\mathrm{S10}, \mathrm{S13}$

S13

$\mathrm{S} 13$

53

S15, S20

$\mathrm{S} 2, \mathrm{~S} 5, \mathrm{S19}, \mathrm{S13}$

S6, 511

S8

S15, S2

$\mathrm{S} 19, \mathrm{~S} 12, \mathrm{S2}, \mathrm{S} 5, \mathrm{~S} 13$

$\mathrm{S} 19, \mathrm{~S} 12$

$\mathrm{S} 12$

$\$ 13$

S18, S7, S15, S19, S12, S2

S8, S20, S11

S15, S19, S12, S5

S19

S2

$\mathrm{S2}$

S2

B. sp. AF14

S8

Capnocytophaga sp.
S2

Chryseobacterium sp. 
Table 3 (continued)

\begin{tabular}{|c|c|c|}
\hline Genus & Species under the corresponding genus & Samples carrying these species \\
\hline \multicolumn{3}{|c|}{ Deinococcus sp. } \\
\hline & D. geothermalis & S6 \\
\hline \multicolumn{3}{|c|}{ Exiguobacterterium sp. } \\
\hline \multicolumn{3}{|c|}{ Gemelia sp. } \\
\hline & G. sanguinis & $\mathrm{S} 3, \mathrm{~S} 8, \mathrm{~S} 2, \mathrm{~S} 14$ \\
\hline & G. morbillorum & S8, S20 \\
\hline \multicolumn{3}{|c|}{ Effusibacillus sp. } \\
\hline \multicolumn{3}{|c|}{ Bacillus sp. } \\
\hline \multicolumn{3}{|c|}{ Planococcus sp. } \\
\hline \multicolumn{3}{|c|}{ Staphylococcus sp. } \\
\hline & S. haemolyticus & S14 \\
\hline & S. simulans & S14 \\
\hline \multicolumn{3}{|c|}{ Aerococcus sp. } \\
\hline & A. vaginalis & $\mathrm{S} 13, \mathrm{~S} 20$ \\
\hline \multicolumn{3}{|c|}{ Dolosigranulum sp. } \\
\hline \multicolumn{3}{|c|}{ Granulicatella sp. } \\
\hline & G. sp. $B B-11$ & S8, S20, S11, S14 \\
\hline & G. sp. canine oral taxon 095 & S14 \\
\hline \multicolumn{3}{|c|}{ Enterococcus sp. } \\
\hline & E. faecium & S16 \\
\hline & E. italicus & S11, S14 \\
\hline & E. xinjiangensis & S16 \\
\hline & E. casseliflavus & S10 \\
\hline & E. hermanniensis & S10 \\
\hline & E. durans & S10 \\
\hline \multicolumn{3}{|c|}{ Lactobacillus sp.* } \\
\hline & L. coryniformis & S11 \\
\hline & L. satsumensis & S14 \\
\hline \multicolumn{3}{|c|}{ Lactococcus sp. } \\
\hline \multicolumn{3}{|c|}{ Streptococcus sp.* } \\
\hline & S. agalactiae & $\mathrm{S} 2, \mathrm{~S} 6, \mathrm{~S} 3, \mathrm{~S} 7, \mathrm{~S} 8, \mathrm{~S} 11, \mathrm{~S} 10, \mathrm{~S} 14$ \\
\hline & S.pneumoniae & S8, S17, S11, S2, S10, S1, S14 \\
\hline & S.parasanguinis & $\mathrm{S} 2, \mathrm{~S} 5, \mathrm{~S} 6, \mathrm{~S} 3, \mathrm{~S} 7, \mathrm{~S} 8, \mathrm{~S} 11, \mathrm{~S} 10, \mathrm{~S} 13, \mathrm{~S} 14, \mathrm{~S} 17, \mathrm{~S} 20$ \\
\hline & S. anginosus & S18, S3, S4, S7, S8, S17, S20, S2, S10, S13, S14 \\
\hline & S. dannielliae & S18, S3, S4, S16, S20, S11, S12, S2, S10 \\
\hline & S. ferus & S18 \\
\hline & S. pantholopis & S3 \\
\hline & S. sp. oral clone ASCB12 & S3, S7, S8, S17, S20, S11, S10, S14 \\
\hline & S. sp. oral taxon GS9 & S3, S11, S14 \\
\hline & S. didelphis & S16 \\
\hline & S. equinus & S4 \\
\hline & S.parasuis & S16 \\
\hline & S. sanguinis & $\mathrm{S} 7, \mathrm{~S} 8, \mathrm{~S} 17, \mathrm{~S} 14$ \\
\hline & S. sp. DD04(2016) & $\mathrm{S7}, \mathrm{S} 11, \mathrm{~S} 14$ \\
\hline & S. sinensis & S7 \\
\hline & S. sp. XJ149-N32 & $\mathrm{S7}, \mathrm{S} 8, \mathrm{~S} 11, \mathrm{~S} 2, \mathrm{~S} 14$ \\
\hline & S. vestibularis & $\mathrm{S7}, \mathrm{S} 8, \mathrm{~S} 17, \mathrm{~S} 11$ \\
\hline & S. mutans & S20 \\
\hline & S. cristatus & $\mathrm{S} 8, \mathrm{~S} 20, \mathrm{~S} 14$ \\
\hline
\end{tabular}


Table 3 (continued)

\begin{tabular}{|c|c|c|}
\hline Genus & Species under the corresponding genus & Samples carrying these species \\
\hline & S. equi & $\mathrm{S} 10, \mathrm{~S} 14$ \\
\hline & S. gordonii & S14 \\
\hline & S. mitis & S14 \\
\hline \multicolumn{3}{|c|}{ Clostridium sp..* } \\
\hline & C. magnum & S1, S2, S3, S4, S15, S17, S18, S19, S20 \\
\hline & C. sp. Marseille & S15, S19, S19, S12 \\
\hline & C. sp. CE6 & S19, S5, S10, S13 \\
\hline & C. sp. CAG:288 & S12 \\
\hline & C. sp. Culture-Jar-56 & S5 \\
\hline \multicolumn{3}{|c|}{ Lutispora sp. } \\
\hline \multicolumn{3}{|c|}{ Fusibacter sp. } \\
\hline \multicolumn{3}{|c|}{ Mogibacterium sp. } \\
\hline \multicolumn{3}{|c|}{ Alkalibacter sp. } \\
\hline \multicolumn{3}{|c|}{ Agathobacter sp. } \\
\hline \multicolumn{3}{|c|}{ Anaerosporobacter sp. } \\
\hline \multicolumn{3}{|c|}{ Anaerostipes sp. } \\
\hline \multicolumn{3}{|c|}{ Blautia sp.* } \\
\hline & B. massiliensis & S18, S19, S9, S20, S2, S5, S10, S13 \\
\hline & B. hydrogenotrophica & $\mathrm{S} 10, \mathrm{~S} 13$ \\
\hline & B. stercosis & S13 \\
\hline \multicolumn{3}{|c|}{ Catonella sp. } \\
\hline \multicolumn{3}{|c|}{ Dorea sp. } \\
\hline & D. formicigenerans & S7, S19, S9, S17, S20, S12, S5, S10 \\
\hline & D. longicatena & S2, S19 \\
\hline \multicolumn{3}{|c|}{ Lachnoclostridium sp. } \\
\hline & [Clostridium]polysaccharolyticum & S15 \\
\hline \multicolumn{3}{|c|}{ Roseburia sp. } \\
\hline & R. hominis & S6, S17, S2, S5 \\
\hline & R. sp. 1120 & S9, S10 \\
\hline \multicolumn{3}{|c|}{ Shuttleworthia sp. } \\
\hline \multicolumn{3}{|c|}{ Stomatobaculum sp. } \\
\hline \multicolumn{3}{|c|}{ Oscilibacter sp. } \\
\hline \multicolumn{3}{|c|}{ Desulfotomaculum sp. } \\
\hline \multicolumn{3}{|c|}{ Peptostreptococcus sp.* } \\
\hline \multicolumn{3}{|c|}{ Faecalibacterium sp.* } \\
\hline & F.prausnitzii & $\mathrm{S} 2, \mathrm{~S} 12, \mathrm{~S} 18, \mathrm{~S} 19, \mathrm{~S} 20$ \\
\hline \multicolumn{3}{|c|}{ Fastidiosipila sp. } \\
\hline \multicolumn{3}{|c|}{ Ruminiclostridium sp. } \\
\hline & Eubacterium siraeum & S18, S19, S20, S12, S13 \\
\hline & Clostridium leptum & S19, S20, S12, S5 \\
\hline \multicolumn{3}{|c|}{ Ruminococcus sp. } \\
\hline & R. sp. CE2 & S16, S15, S19, S2, S10, S13 \\
\hline & R. sp. YE58 & $\mathrm{S} 15, \mathrm{~S} 9, \mathrm{~S} 10$ \\
\hline & R. sp. RLB3 & S9, S12 \\
\hline & R. sp. W22 & S9, S17, S10, S13 \\
\hline & R. sp. 653 & S17 \\
\hline & R. bromii & $\mathrm{S} 12, \mathrm{~S} 5$ \\
\hline & R. sp. YE281 & $\mathrm{S} 12$ \\
\hline & R. sp. ID1 & S2 \\
\hline
\end{tabular}


Table 3 (continued)

\begin{tabular}{|c|c|c|}
\hline Genus & Species under the corresponding genus & Samples carrying these species \\
\hline & R. gauvreaucii & S5 \\
\hline & R. sp. Marseille-P328 & S13 \\
\hline \multicolumn{3}{|c|}{ Subdoligranulum sp. } \\
\hline \multicolumn{3}{|c|}{ Dethiobacter sp. } \\
\hline \multicolumn{3}{|c|}{ Catenibacterium sp. } \\
\hline & C. mitsuokai & S18, S7, S15, S19, S9, S5, S17 \\
\hline \multicolumn{3}{|c|}{ Holdemanella sp.* } \\
\hline \multicolumn{3}{|c|}{ Solobacterium sp. } \\
\hline \multicolumn{3}{|c|}{ Turicibacter sp. } \\
\hline \multicolumn{3}{|c|}{ Phascolarctobacterium sp. } \\
\hline & P. sp. 377 & S17 \\
\hline & P. sp. canine oral taxon 212 & $\mathrm{~S} 17, \mathrm{~S} 5$ \\
\hline \multicolumn{3}{|c|}{ Megamonas sp. } \\
\hline \multicolumn{3}{|c|}{ Dialister sp.* } \\
\hline & D. sp. 67 & $\mathrm{~S} 18, \mathrm{~S} 8, \mathrm{~S} 15, \mathrm{~S} 19, \mathrm{~S} 9, \mathrm{~S} 17, \mathrm{~S} 20, \mathrm{~S} 12, \mathrm{~S} 2, \mathrm{~S} 10, \mathrm{~S} 13$ \\
\hline & D. succinatiphilus & S17 \\
\hline \multicolumn{3}{|c|}{ Veillonella sp. } \\
\hline & V. sp. oral clone OH1A & S3, S8, S20, S10, S13.S14 \\
\hline & V. sp. oral taxon 780 & $\mathrm{~S} 8, \mathrm{~S} 2, \mathrm{~S} 13$ \\
\hline & V.atypica & $\mathrm{S} 17, \mathrm{~S} 13$ \\
\hline & V. sp. 2011-11eoVSA-F2 & S17 \\
\hline & V.magna & S20 \\
\hline \multicolumn{3}{|c|}{ Anaerococcus sp. } \\
\hline & A. sp. $S 138$ & S4 \\
\hline & A. prevotii & S16 \\
\hline & A. sp. $S 194$ & S16 \\
\hline & A. octavius & S7 \\
\hline \multicolumn{3}{|c|}{ Peptoniphilus sp. } \\
\hline \multicolumn{3}{|c|}{ Fusobacterium sp. } \\
\hline & F. nucleatum & S11 \\
\hline & F. russii & S2 \\
\hline \multicolumn{3}{|c|}{ Leptotricha sp. } \\
\hline & L. sp. oral clone FP036 & S11 \\
\hline & L. sp. oral taxon 212 & S11, S2, S14 \\
\hline & L. sp. oral taxon 847 & $S 11, S 2$ \\
\hline & L. buccalis & S14 \\
\hline \multicolumn{3}{|c|}{ Streptobacillus sp. } \\
\hline & S. hongkonggensis & S6, S20 \\
\hline \multicolumn{3}{|c|}{ Brevundimonas sp. } \\
\hline \multicolumn{3}{|c|}{ Methylobacterium sp.* } \\
\hline \multicolumn{3}{|c|}{ Paracoccus sp. } \\
\hline \multicolumn{3}{|c|}{ Actererythrobacter sp. } \\
\hline \multicolumn{3}{|c|}{ Novosphingobium sp. } \\
\hline \multicolumn{3}{|c|}{ Sphingomonas sp. } \\
\hline \multicolumn{3}{|c|}{ Lautropia sp. } \\
\hline & L. mirabilis & $\mathrm{S} 2, \mathrm{~S} 6, \mathrm{~S} 11, \mathrm{~S} 13, \mathrm{~S} 14$ \\
\hline \multicolumn{3}{|c|}{ Ralstonia sp. } \\
\hline Parasutte & & \\
\hline & P. excrementihominis & S10 \\
\hline
\end{tabular}


Table 3 (continued)

\begin{tabular}{|c|c|c|}
\hline Genus & Species under the corresponding genus & Samples carrying these species \\
\hline \multicolumn{3}{|c|}{ Sutterella sp. } \\
\hline & S. sp. YIT-12072 & S7, S5 \\
\hline \multicolumn{3}{|c|}{ Neisseria sp.* } \\
\hline & N. meningitidis & $\mathrm{S} 18, \mathrm{~S} 19, \mathrm{~S} 20$ \\
\hline & N. shayeganii & $\mathrm{S} 18, \mathrm{~S} 19, \mathrm{~S} 20$ \\
\hline & N. elongata & $\mathrm{S} 11, \mathrm{~S} 14$ \\
\hline & N. flavescens & S11 \\
\hline & N. lactamica & S2 \\
\hline \multicolumn{3}{|c|}{ Simonsiella sp. } \\
\hline \multicolumn{3}{|c|}{ Campylobacter sp. } \\
\hline & C. faecalis & S18 \\
\hline & C. concisus & S11 \\
\hline \multicolumn{3}{|c|}{ Helicobacter sp. } \\
\hline & H.pylori & S2, S6, S7, S11, S14, \\
\hline \multicolumn{3}{|c|}{ Shewanella sp. } \\
\hline \multicolumn{3}{|c|}{ Rheinheimera sp. } \\
\hline \multicolumn{3}{|c|}{ Buttiauxella sp. } \\
\hline \multicolumn{3}{|c|}{ Candidatus Benitsuchiphilus sp. } \\
\hline \multicolumn{3}{|c|}{ Candidatus Blochmannia sp. } \\
\hline \multicolumn{3}{|c|}{ Citrobacter sp. } \\
\hline & C. amalonaticus & S10 \\
\hline \multicolumn{3}{|c|}{ Eschericia sp. } \\
\hline & E. coli & $\mathrm{S} 1, \mathrm{~S} 2, \mathrm{~S} 3, \mathrm{~S} 4, \mathrm{~S} 6, \mathrm{~S} 8, \mathrm{~S} 10, \mathrm{~S} 11, \mathrm{~S} 13,16, \mathrm{~S} 17, \mathrm{~S} 19, \mathrm{~S} 20$ \\
\hline & E. albertii & $\mathrm{S} 1, \mathrm{~S} 2, \mathrm{~S} 4, \mathrm{~S} 6, \mathrm{~S} 10, \mathrm{~S} 11, \mathrm{~S} 13, \mathrm{~S} 20$ \\
\hline & E.sp. & S1, S11 \\
\hline \multicolumn{3}{|c|}{ Klebsiella sp. } \\
\hline & K. sp. A4 & $\mathrm{S} 1, \mathrm{~S} 2, \mathrm{~S} 6, \mathrm{~S} 13, \mathrm{~S} 18$ \\
\hline & K. pneumoniae & $\mathrm{S} 4, \mathrm{~S} 7, \mathrm{~S} 10$ \\
\hline & K. aerogenes & S7 \\
\hline \multicolumn{3}{|c|}{ Shigella sp. } \\
\hline & S. dysenteriae & S6, S11 \\
\hline & S. sonnei & S6, S10, S16 \\
\hline \multicolumn{3}{|c|}{ Shimwella sp. } \\
\hline \multicolumn{3}{|c|}{ Xenorhabdus sp. } \\
\hline \multicolumn{3}{|c|}{ Brenneria sp. } \\
\hline & B. sp. DAFNE_Bnig-1 & S4 \\
\hline & B. populi & $\mathrm{S} 10$ \\
\hline \multicolumn{3}{|l|}{ Dickeya } \\
\hline \multicolumn{3}{|c|}{ Serratia sp } \\
\hline & S. marcescens & S3, S11 \\
\hline \multicolumn{3}{|c|}{ Alcanivorax sp. } \\
\hline \multicolumn{3}{|c|}{ Halomonas sp. } \\
\hline \multicolumn{3}{|c|}{ Aggregatibacter sp. } \\
\hline \multicolumn{3}{|c|}{ Haemophilus sp. } \\
\hline & H. haemolyticus & $\mathrm{S} 4, \mathrm{~S} 6, \mathrm{~S} 8$ \\
\hline & H. parainfluenzae & $\mathrm{S} 4, \mathrm{~S} 8, \mathrm{~S} 14$ \\
\hline & H. pittmaniae & S8 \\
\hline & H. sp. oral clone BJ021 & S17 \\
\hline & H. sputorum & $\mathrm{S} 11, \mathrm{~S} 17$ \\
\hline
\end{tabular}


Table 3 (continued)

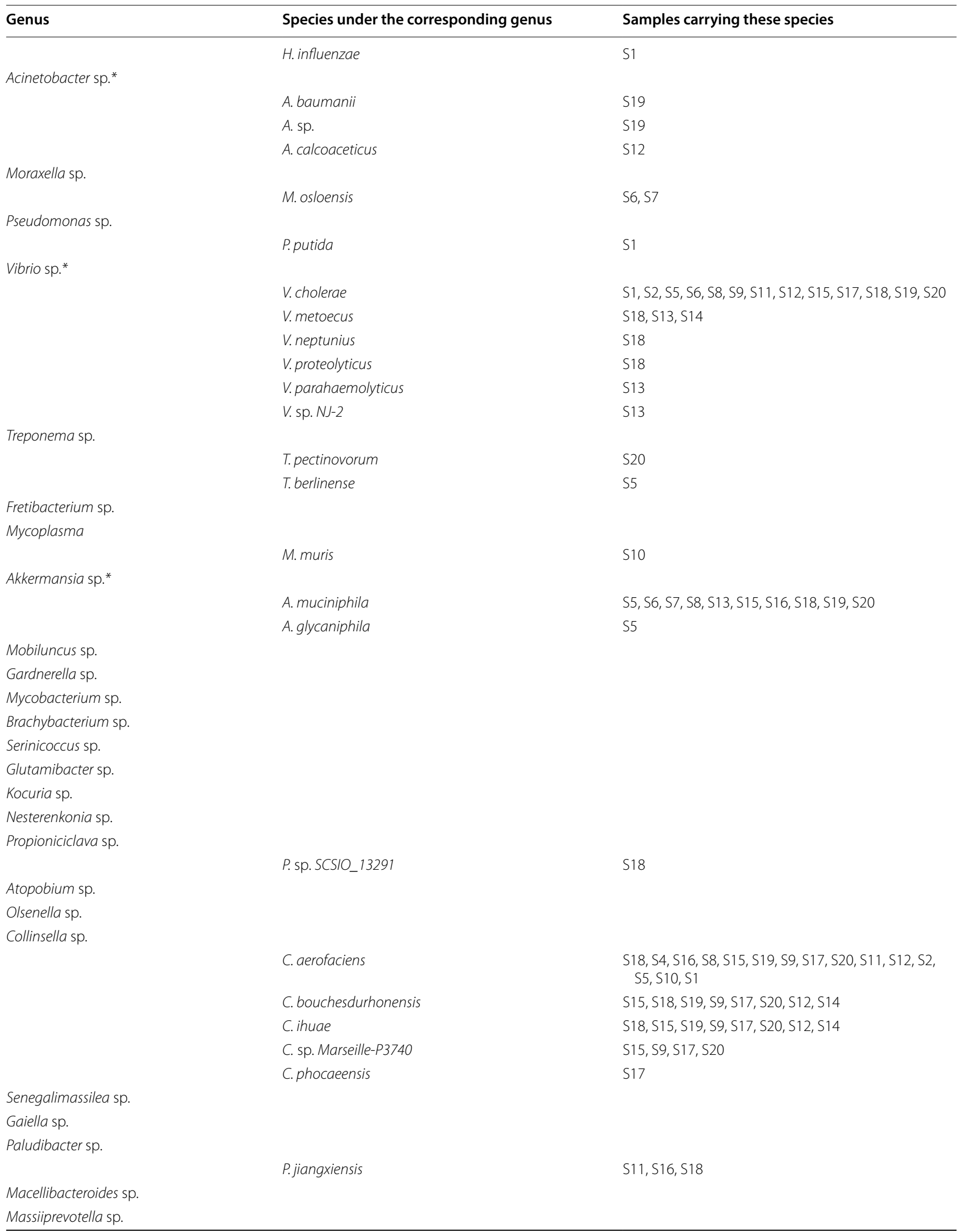


Table 3 (continued)

\begin{tabular}{|c|c|c|}
\hline Genus & Species under the corresponding genus & Samples carrying these species \\
\hline & M. massiliensis & $\mathrm{S} 1, \mathrm{~S} 2, \mathrm{~S} 5, \mathrm{~S} 9, \mathrm{~S} 12, \mathrm{~S} 18, \mathrm{~S} 19, \mathrm{~S} 20$ \\
\hline \multicolumn{3}{|c|}{ Prevotellamassilia sp } \\
\hline & P. timonensis & $\mathrm{S} 18, \mathrm{~S} 19, \mathrm{~S} 17, \mathrm{~S} 20, \mathrm{~S} 12, \mathrm{~S} 2, \mathrm{~S} 10$ \\
\hline \multicolumn{3}{|c|}{ Alistipes sp. } \\
\hline & A. finegoldii & S15 \\
\hline \multicolumn{3}{|c|}{ Empedobacter sp. } \\
\hline \multicolumn{3}{|c|}{ Arcticibacter sp. } \\
\hline \multicolumn{3}{|c|}{ Sphingobacterium sp. } \\
\hline & S. sp. JAS3 & S19, S20 \\
\hline \multicolumn{3}{|c|}{ Microcystis sp. } \\
\hline & M. sp. SAG43. 90 & S18 \\
\hline \multicolumn{3}{|c|}{ Elusimicrobium sp. } \\
\hline & E. minutum & S12 \\
\hline \multicolumn{3}{|c|}{ Fibrobacter sp. } \\
\hline \multicolumn{3}{|c|}{ Lactobacillalis bacterium HY-36-1) } \\
\hline \multicolumn{3}{|c|}{ Weissella sp. } \\
\hline \multicolumn{3}{|c|}{ Howardella sp. } \\
\hline & H. ureilytica & S2 \\
\hline \multicolumn{3}{|c|}{ Intestimonas sp. } \\
\hline \multicolumn{3}{|c|}{ Christensenella sp. } \\
\hline & C. massiliensis & S5, S19 \\
\hline \multicolumn{3}{|c|}{ Butyricoccus sp. } \\
\hline & B. faecihominis & S9, S17, S20, S13 \\
\hline \multicolumn{3}{|c|}{ Oxobacter sp. } \\
\hline \multicolumn{3}{|c|}{ Acidaminobacter sp. } \\
\hline \multicolumn{3}{|c|}{ Anaerofustis sp. } \\
\hline \multicolumn{3}{|c|}{ Eubacterium sp. } \\
\hline & E. coprostanoligenes & $\mathrm{S} 18, \mathrm{~S} 19, \mathrm{~S} 20$ \\
\hline & E. eligens & $\mathrm{S} 2, \mathrm{~S} 5, \mathrm{~S} 10, \mathrm{~S} 12, \mathrm{~S} 17, \mathrm{~S} 18, \mathrm{~S} 19, \mathrm{~S} 20$ \\
\hline & E. sp. oral clone DO 016 & $\mathrm{~S} 3, \mathrm{S7}, \mathrm{S} 8$ \\
\hline & E. sp. oral clone FX028 & S7, S14 \\
\hline & E.pyruvativorans & S12 \\
\hline & E. sp. oral clone El074 & $\mathrm{S} 14$ \\
\hline
\end{tabular}

Butyrivibrio
B. crossotus
$\mathrm{S} 12, \mathrm{~S} 18, \mathrm{~S} 19, \mathrm{~S} 20$

Eisenbergiella sp.

Fusicatenibacter sp.

Lachnospira sp.

Marvinbryantia sp.

Moryella sp.

Oribacterium sp.

Tyzzerella sp.

Peptococcus sp.

Romboutsia sp.

Candidatus soleaferrea

Fournierella sp.

Negativibacillus sp.

Papillibacter sp. 
Table 3 (continued)

\section{Genus}

Phoceasp.

Saccharofermentans sp.

sporobacter sp.

Dielma sp.

Erysipelothrix sp.

Anaerovibrio sp.

Mitsuokella sp.

Selemonas sp.

$$
\text { S. sputigena }
$$

S. noxia

\section{A. histaminiformans}

M. micronuciformis

Finegoldia sp.

Parvimonas sp.

Cetobacterium sp.

Craurococcus sp.

Roseomonas sp.

Candidatus Alysiosphaera

sphaerotilus sp.

Comamonas sp.

Diaphorobacter sp.

Oxalobactersp.

$$
\begin{aligned}
& \text { C. aquatica } \\
& \text { C. testosteroni }
\end{aligned}
$$

O. formigenes

Eikenella sp.

Kingella sp.

Thiobacillus sp.

Azonexus sp.

Zoogloea sp.

Desulfomicrobium sp.

$$
\text { D. baculatum }
$$

Bilophila sp.

Desulfovibrio sp.

$$
\begin{aligned}
& \text { D. putealis } \\
& \text { D. sp. enrichment clone JdgSrb011 }
\end{aligned}
$$

Mailhella sp.

$$
\text { M. massiliensis }
$$

$\mathrm{S} 12$

$\mathrm{S} 18, \mathrm{~S} 19, \mathrm{~S} 10$

S18, S3, S4, S15, S19, S17, S20, S2, S5, S1, S14

S3, S4, S15, S17, S2, S1, S14

$\mathrm{S} 11$

$\mathrm{S} 18, \mathrm{~S} 19, \mathrm{~S} 20, \mathrm{~S} 12$

Pelobacter sp

Geobacter sp.

Arcobacter sp.

Sulfurospirillum sp. 
Table 3 (continued)

Genus

Aeromonas sp.

Anaerobiospirillum sp.

Ruminobacter sp.

Succinivibrio $s p$

Plesiomonas sp

Cronobacter sp.

Buchnera sp.

Edwardsiella sp.

Morganella sp.

Methyloparacoccus sp.

Actinobacillus sp.

Aliivibrio sp.

Lysobacter sp.

Stenotrophomonas sp.

Bacteriovorax sp.

Peredibacter sp.

Brachyspira sp.

Asteroleplasma sp.

Gordonia sp.

Tessaracoccus sp.

Candidatus Saccharimonas

Listeria sp.

Facklamia sp.

Ignavigranum sp.

Melissococcus sp.

Pilibacter sp.

Leuconostoc sp.

Epulopiscium sp.

Anaerovorax sp.

Johnsonella sp.

Clostridioides

Halanaerobium sp.

Anaeroglobus sp.

Murdochiella sp.

Ignavibacterium sp.

Magnetococcus sp.

Vogesella sp.

Marinobacter sp.

Franconibacter sp.

\section{J. ignava}

C. difficile
L. floridensis

A. defectiva

Samples carrying these species

$\mathrm{S} 2, \mathrm{~S} 17, \mathrm{~S} 20$

$\mathrm{S} 18, \mathrm{~S} 19$

$\mathbf{S 1 8}$

B. aphidicola

S16

$\mathrm{S1} 1, \mathrm{S2}, \mathrm{S} 3, \mathrm{~S} 8, \mathrm{~S} 17, \mathrm{~S} 20, \mathrm{~S} 11, \mathrm{~S} 13, \mathrm{~S} 14$
S14

S3, S8

Pantoea sp. 
Table 3 (continued)

Genus

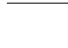

(2)

Coxiella sp.

Ureaplasma sp.

Varibaculum sp.

Lawsonella sp.

L. clevelandensis

S7

Williamsia sp.

Arthrobacter sp.

Saccharopolyspora sp.

Flavobacterium sp.

F.caeni

S2

Moheibacter sp.

Alkaliphilus sp.

Acetobacterium sp.

A. sp. LacT

S11

A. woodii

S11

Desulfosporosinus sp.

Anaerotruncus sp.

Negativicoccus sp.

Fimbriiglobus sp.

Haliangium sp.

Enterobacter sp.

E. cloacae

$\mathrm{S} 10$

E. hormaechei

S1

Salmonella sp.

S. enterica

S11

E. teleogrylli

S16

P. mirabilis

S14

Sodalis sp.

Hahella sp.

Phocoenobactersp.

Perlucidibaca sp.

Luteimonas sp.

Arcella sp.

Bryobactersp.

Aurantimicrobium sp.

Huakuichenia sp.

Propionimicrobium sp.

Emticicia sp.

Cloacibacterium sp.

Salinicoccus sp.

Proteiniclasticum sp.

Ethanoligenens sp. 
Table 3 (continued)

\section{Genus}

Ezakiella sp.

Gallicola sp.

Helcococcus sp.

Limnobacter sp

Dechloromonas sp.

Propionivibrio sp.

Acidibacter sp

Nocardia sp.

Pseudopropionibacterium sp.

Barnesiella sp.

Culturomica sp.

Hydrogenispora sp.

Atopococcus sp.

Catellicoccus sp.

Fenollaria sp.

Neofamilia sp.

Sarcina sp.

Acetoanaerobium sp.

Bulleidia sp.

Candidatus Stoquefichens

Coprobacillus sp.

Erysipelatoclostridium sp.

Sedimentibacter sp.

Aureimonas sp.

Aquabacterium sp.

Paraburkholderia sp.

Massilia sp.

Candidatus Babela

Cardiobacterium sp.

Legionella sp.

Bdellovibrio sp.

Illumatobacter sp

Acidothermus sp.

Haloactinopolyspora sp.

Quadrisphaera sp.

Georgenia sp.

Cellulomonas sp.

Dermabacter sp.

Pseudoclavibacter sp.

Nocardioides sp.

Eggerthella sp.

Roultibacter sp.
B. extructa

58

Species under the corresponding genus

Samples carrying these species

H. sueciensis

S7

S7

S7

B. sp. SRP1

59

R. timonensis 
Table 3 (continued)

\section{Genus}

Rubrobacter sp.

Dysgonomonas sp.

Tannerella sp.

Asinibacterium sp.

Sediminibacterium $\mathrm{sp}$.

Marinoscilum sp.

Neochlamydia sp.

Paenibacillus sp.

Eremococcus sp.

Lacticigenium sp.

Tetragenococcus sp.

Vagococcus sp.

Lachnoanaerobaculum sp.

Filibacter sp.

Eggerthia sp.

Hyphomicrobium sp.

Candidatus Ovatusbacter sp

Cellvibrio sp.

Yersinia sp.

Psychrobacter sp.

Pseudoxanthomonas sp.

Thermomonas sp.

Candidatus Ancillula sp.

Aeriscardovia sp.

Pseudoscardovia sp.

Candidatus Aquiluna sp.

Candidatus Limnoluna sp.

Haematomicrobium sp.

Nakamurella sp.

Dinghuibacter sp.

Pasteuria sp.

Alloiococcus sp.

Flavonifractor sp.

Caloranaerobacter sp

Caminicella sp.

Syntrophococcus sp.

Coprothermobacter sp.

Singulisphaera sp.

Pirellula sp.

Plantoptrus sp.

Devosia sp.

Amaricoccus sp.

Reyranella sp.
Species under the corresponding genus

Samples carrying these species

T. forsythia

$\mathrm{S} 8, \mathrm{~S} 2, \mathrm{~S} 1$

S. sp. LT21-MRL

S8

F. alocis

S14

E. catenaformis

S8, S14

$\mathrm{S} 8, \mathrm{~S} 17, \mathrm{~S} 20, \mathrm{~S} 11, \mathrm{~S} 10, \mathrm{~S} 14$ 
Table 3 (continued)

\section{Genus}

Thiomonas sp.

Undibacterium sp.

Duodenibacillus sp.
Samples carrying these species

Species under the corresponding genus

H. foetida

Acidimicrobium sp.

Cellulosimicrobium sp.

Rikenella sp.

Marinifilum sp.

Candidatus Latescibacter sp.

Trichococcus sp.

Colidextribacter sp.

Proteiniborus sp.

Caloramator sp.

Hungatella sp.

Acetitomaculum sp.

Cellulosilyticum sp.

Herbinix sp.

Robinsoniella sp.

Sellimonas sp.

Peptoclostridium sp.

Acetivibrio sp.

Anaerofilum sp.

Hydrogenoanaerobacterium sp.

Oscillospira sp.

Catenisphaera sp.

Dubosiella sp.

Schwartzia sp.

Gottschalkia sp.

Victivallis sp.

Candidatus Paracaedibacter sp.

Enhydrobacter sp.

Komagataeibacter sp.

Ideonella sp.

Acidovorax sp.

Alicycliphilus sp.

Delftia sp.

Alysiella sp.

Vitreoscilla sp.
$\mathrm{S} 12$ 
Table 3 (continued)

\section{Genus}

Dechlorobacter sp.

Azoarcus sp.

Thauera sp.

Geothermobacter sp.

Succinatimonas sp.

Pseodoalteromonas sp.

Psychromonas sp.

Woeseia sp.

Candidatus Rosenkranzia

Marinomonas sp.

Alkanindiges sp.

Candidatus Parabeggiatoa sp.

Dokdonella sp.

Piscicoccus sp.

Agromyces sp.

Sanguibacter $\mathrm{sp}$.

Sanguibacteroides sp.

Enorma sp.

Paraeggerthella sp.

Nubsella sp.

Pedobacter sp.

Caldicoprobacter sp

Lactonifactor sp.

Coprococcus sp.

$$
\text { C. catus }
$$

P. sp. CA38

Faecalibaculum sp.

Faecalitalea sp.

Merdibacter sp.

Gemmatirosa sp.

Microvirga sp.

Candidatus Thiosymbion sp.

Oblitimonas sp.

Alloscardovia sp.

Leucobacter sp.

Longispora sp.

Acetatifactor sp.

Cryptanaerobacter sp.

Breznakia sp.

Succiniclasticum sp.

Sneathia sp.

Kaistia sp.

Hellea sp.

Pseudoruegeria sp. 
Table 3 (continued)

\section{Genus}

Candidatus Accumulibacter sp.

Rhodoferax sp.

Anaeromyxobacter sp.

Mannheimia sp.

M. haemolytica

S17

Mesocricetibacter sp.

Fluviicoccus sp.

Photobacterium sp.

Dermacoccus sp.

Dactylosporangium sp.

Propionibacterium sp.

Coriobacterium sp.

Cryptobacterium sp.

Gordonibacter sp.

Phocoeicolasp.

Rufibacter sp.

Truepera sp.

Desulfuribacillus sp.

Oceanobacillus sp.

Sporosarcina sp.

Marinilactibacillus sp.

M. sp. G13. 51

Candidatus Arthromitus sp.

Guggenheimella sp.

Proteocatella sp.

Terrisporobacter sp.

Quinella sp.

Acidaminococcus sp.

Anaerospora sp.

Dethiosulfatibacter sp

Tissierella sp.

Asticcacaulis sp.

Sphingobium sp.

tepidomonas sp.

Halioglobus sp.

Candidatus Stammerula sp.

Izhakiella sp.

Pectobacterium sp.

Aquicella sp.

Scardovia sp.

Luedemannella sp.

Rosemarinus sp.

Maritimimonas sp.

Bavariicoccus sp.

$$
\text { B. seileri }
$$

Bradyrhizobium sp.

Rhizobium sp.

Burkholderia sp. 
Table 3 (continued)

\section{Genus}

Candidatus Glomeribacter sp

Polynucleobacter sp.

Curvibacter sp.

Conchiformibius sp.

Snodgrassella sp.

Stenoxybacter sp.

Tolumonas sp.

Idiomarina sp.

Photorhabdus sp.

Chromohalobacter sp

Balneatrix sp.

Oleispira sp.

Galeibacterium sp.

Pasteurella sp.

Anaerocella sp.

Myroides sp.

Jeotgalicoccus sp.

Pseudoflavonifractor sp.

Thermobrachium sp.

Mobilitalea sp.

Marseilibacter sp.

Paeniclostridium sp.

Anaerobacterium sp.

Holdemania sp.

Pedomicrobium sp.

Rubellimicrobium sp.

Rivibacter sp.

Alcaligenes sp.

Sulfurimonas sp.

Pseudohongiella sp.

Zobellella sp.

Thiorhodovibrio sp.

Providencia sp.

neptunomonas sp.

Azotobacter sp.

Thiopseudomonas sp.

Beggiatoa sp.

Arenimonas sp.

Anaeroplasma sp.

Chlamydia sp.

Pectinatus sp.

Candidatus Gullanella sp.

Orbus sp.

achromatium sp.

Fermentimonas sp.

Nitribacter sp.

Natranaerovirga sp.

Species under the corresponding genus

P. muttocida

S11

Samples carrying these species 
Table 3 (continued)

\section{Genus}

Parasporobacterium sp.

Acetanaerobacterium sp.

Angelakisella sp.

Ercella sp.

Pygmaiobacter sp.

Thermodesulfovibrio sp.

Caulobacter sp.

Candidatus hamiltonella sp.

Pyramidobacter sp.

Luteipulveratus sp.

Aestuarlimicrobium sp.

Spirosoma sp.

Acidibacillus sp.

Sinibacillus sp.

Solibacillus sp.

Carnobacterium sp.

Constrictibacter sp.

Endozoicomonas sp.

Kistimonas sp.

Oleiphilus sp.

Synergistes $\mathrm{sp}$.

Ornithinimicrobium sp.

Pseudonocardia sp.

Phoenicibacter sp.

Cytophagasp.

Alsobacter sp.

Rhodobacter sp.

Magnetospira sp.

Paraglaciecola sp.

Candidatus Purcelliella sp.

Kosakonia sp.

Candidatus Schmidhempelia sp.

Parviterribacter sp.

Haoranjiania sp.

Hathewaya sp.

Acidiphilum sp.

Sulfurirhabdus sp.

Candidatus Regiella sp.

Ammoniibacillus sp.

Macrococcus sp.

Thermoactinomyces sp.

Jeotgalibaca sp.

Phenylobacterium sp.

Cupriavidus sp.

Methyloversatalis sp.

\section{Species under the corresponding genus}

Samples carrying these species

P. paucivorans

S5

A. massiliensis

S5

A. kwangyangense

P. pentastirinorum

S1 
Table 3 (continued)

\begin{tabular}{l}
\hline Genus $\quad$ Species under the corresponding genus \\
\hline Desulfuromonas sp. \\
Ferrimonas sp. \\
Vulcaniibacterium sp.
\end{tabular}

Genus marked with an * were found in all the twenty diarrheal samples

mean abundance between the different families in diarrheal samples. Mean abundance of Bifidobacteriaceae was found to be lower than that of Enterobacteriaceae and Vibrionaceae, however the two-tailed p-values were non significant at 0.2571 and 0.3683 and median values of 1.1 and -0.1750 respectively. Mean abundance of Lachnospiraceae was found to be lower than that of Enterobacteriaceae and the two-tailed p-value was non significant at 0.5412 and median of -0.9 . Mean abundance of Lachnospiraceae was significantly lower than that of Vibrionaceae with two-tailed p-value of 0.0233 and median was 1.240. Mean abundance of Enterobacteriaceae was found to be significantly higher than that of Aeromonadaceae with a two-tailed p-value of $<0.0001$ and median of -3.199 but non-significantly higher than that of Vibrionaceae with two-tailed p-value of 0.0711 and median of -2.640 .

\section{Difference in abundance of commensals and pathogens in diarrheal samples confirmed with Vibrio sp. infection} Wilcoxon matched-pairs signed rank test was used to compare the difference in mean abundance of Vibrionaceae and Bifidobacteriaceae, Vibrionaceae and Lachnospiraceae, Vibrionaceae and Enterobacteriaceae in samples from which Vibrio sp. was isolated as the etiologic agent of diarrhea. Mean abundance of Vibrionaceae was found to be higher than that of Bifidobacteriaceae and Lachnospiraceae but the difference was non-significant with two-tailed p-values of 0.5186 and 0.5703 and median of 0.07000 and -0.05500 respectively. Mean abundance of Vibrionaceae was found to be lower than that of Enterobacteriaceae but the difference was nonsignificant with two-tailed p-values of 0.5693 and median of -1.405 . These results have been depicted in Fig. 6c.

\section{Difference in abundance of commensals and pathogens in diarrheal samples confirmed with Aeromonas sp. infection}

Unpaired t-test with Welch's correction was used to compare the difference in mean abundance of Aeromonadaceae with that of Bifidobacteriaceae, Lachnospiraceae, and Enterobacteriaceae in samples S15 and S16 from which Aeromonas sp. was isolated as the etiologic agent of diarrhea (Table 1). Mean abundance of Aeromonadaceae was found to be lower than that of Bifidobacteriaceae, Lachnospiraceae and Enterobacteriaceae, but the difference was non-significant with two-tailed p-values of $0.3476,0.4938,0.4298$ respectively. These results have been represented in Fig. $6 \mathrm{~d}$.

\section{Statistical analysis of Bacteroidetes/Firmicutes ratio}

The Bacteroidetes/Firmicutes $(\mathrm{B} / \mathrm{F})$ ratio was calculated to predict dysbiosis related to diarrhea. $\mathrm{B} / \mathrm{F}$ ratio obtained was in the range of 0.001056943 to 1.536455818 (Table 1 and Fig. 7) with a median ratio of 0.11 and a mean ratio of 0.407702313 . The standard deviation was \pm 0.454603761 . The normal Distribution Curve showed $68 \%$ of diarrheal population has a $\mathrm{B} / \mathrm{F}$ ratio of 0.86 to $0.05,95 \%$ has a ratio of 1.32 to 0.50 and $99.7 \%$ has a ratio of 1.77 to 0.96 . The $\mathrm{z}$-score ranged between -0.33 and 2.48 standard deviations of the mean value. In all samples except S13 and S15, abundance of Firmicutes exceeded that of Bacteroidetes.

Samples were grouped according to various parameters like age, sex, diarrheal etiology and residential location (urban or suburban). Difference in $\mathrm{B} / \mathrm{F}$ ratio between these groups was compared and significance of the difference determined by unpaired t-test. Difference of $\mathrm{B} / \mathrm{F}$ ratio between male and female of age $\geq 2$ years was found. Mean $\mathrm{B} / \mathrm{F}$ ratio of male was 0.4 and that of female was 0.3. However, this difference was found to be non-significant, Difference of mean $\mathrm{B} / \mathrm{F}$ ratio between samples with single diarrheal pathogen and two pathogens was non-significant. Mean B/F ratio of samples from urban areas and samples from suburban areas were 0.4 and 0.5 respectively and the difference was non-significant. Analysis of variance (ANOVA) was performed to determine the significance of $\mathrm{B} / \mathrm{F}$ ratio among samples of three age groups, 0-5 years, 5-15 years and > 15 years and was found non-significant. One sample $t$ and Wilcoxon test was performed on $\mathrm{B} / \mathrm{F}$ ratios of samples associated with $V$. cholerae (VC) infection and non-VC infections. The first group was significant with two-tailed p-value of $<0.0001$. 


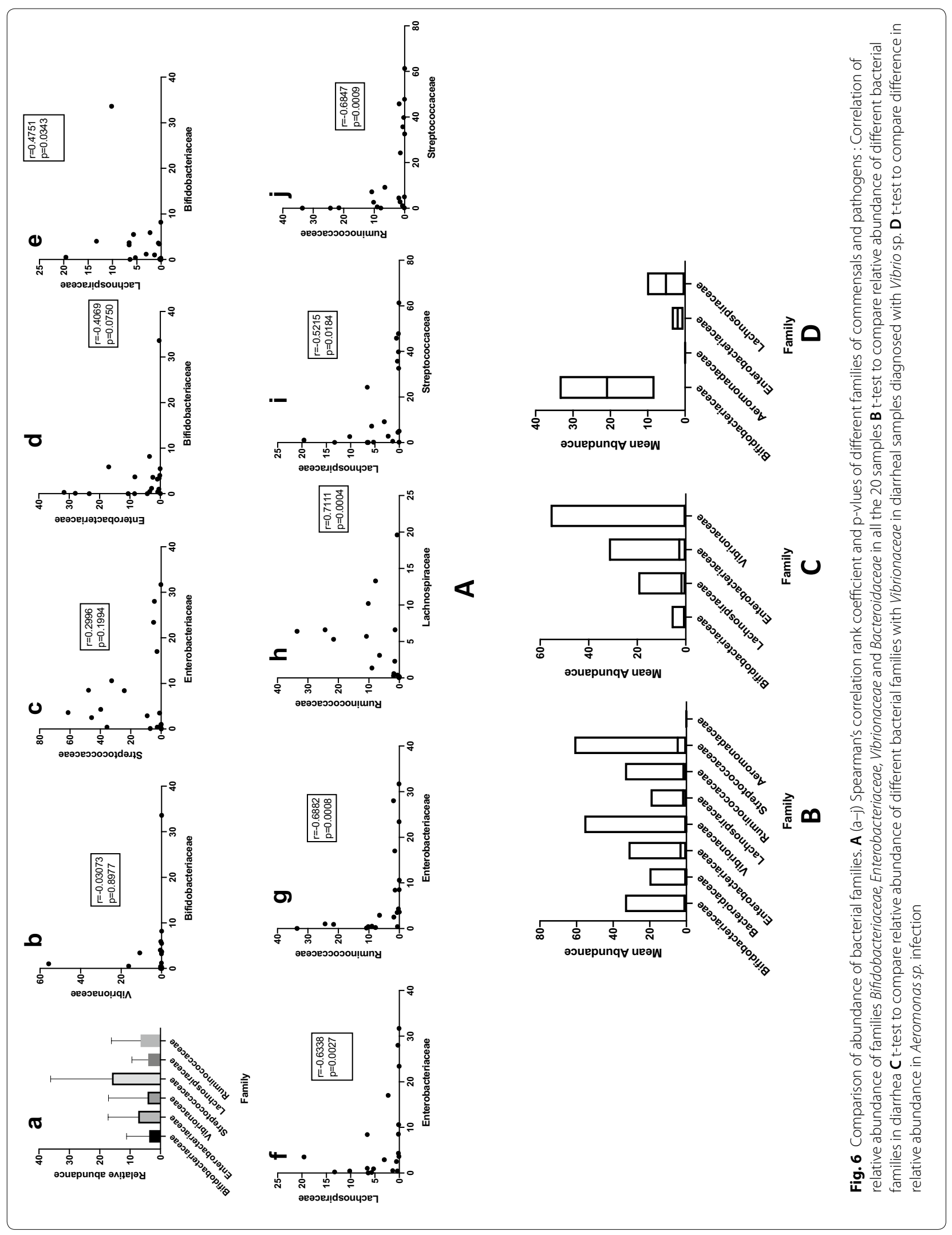




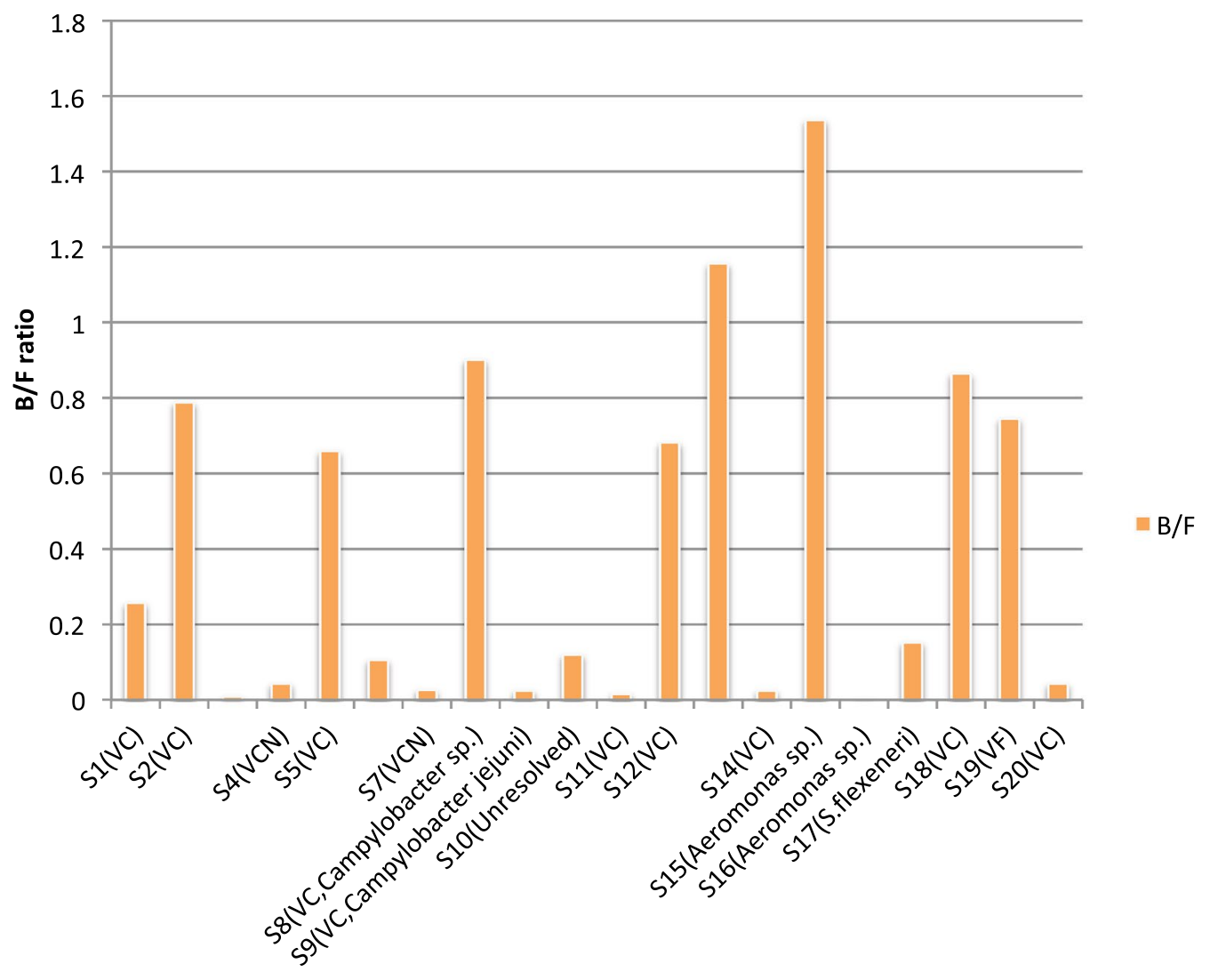

Samples

Fig. 7 B/F ratio in diarrheal samples. Bacteroidetes/Firmicutes ratio in diarrheal samples shows a ratio of $<1$ in all the samples except S13 and S15. The diarrheal agent isolated from the sample has been indicated in parenthesis beside each sample

\section{Alpha and beta diversity}

Alpha diversity ( $\alpha$-diversity) is used to study the richness and evenness of species diversity within a sample while beta-diversity ( $\beta$-diversity) is used to calculate the species diversity between two samples. Therefore, $\alpha$-diversity was calculated to understand OTU diversity, richness and evenness within each of the 20 diarrheal samples and represented by Shannon-index while $\beta$-diversity was used to compare OTU diversity among these twenty samples. Figure 8 shows the $\alpha$-diversity observed among the diarrheal samples. The samples could be sequenced to a variable range of depth of $2 e+05$ to $5 \mathrm{e}+05$ and showed variable evenness and richness of microbial diversity among them even if two samples were associated with the same diarrheal pathogen. S20 had the highest $\alpha$-diversity while S1 had the lowest although VC O1 was isolated from both. From S8, S13, S14 different diarrheal pathogens were isolated but they showed the same Shannon index indicating the same level of richness and evenness of OTUs.
Figure 9 represents the $\beta$-diversity among the samples. The PCA (Principal Component Analysis) shows that each sample is unique and has variable OTU diversity and OTU abundance compared to one another even if the stool samples were associated with the same diarrheal pathogen. The axis $\mathrm{PC} 1$ was more informative than PC2 about the $\beta$-diversity. The samples were divided into six groups based on etiologic agent of diarrhea isolated from the stool by culture method and in the Fig. 9 samples were represented with a different colour to indicate the group it belongs to. These six groups are co-infection (CI), V.cholerae (VC), other Vibrio (O_V) and VC nonO1/non O139 (VCN). Samples S3, S6, S8 and S9 associated with $\mathrm{CI}$ did not cluster together indicating they have different $\beta$-diversity, similar trend was found in S4 and S7, associated with VCN. S12 associated with VC and S19 associated with other Vibrio sp.were closer although they were associated with different etiologic agents of diarrhea.

The heat map in Fig. 10 was constructed to represent the relative abundance of various bacterial families in 


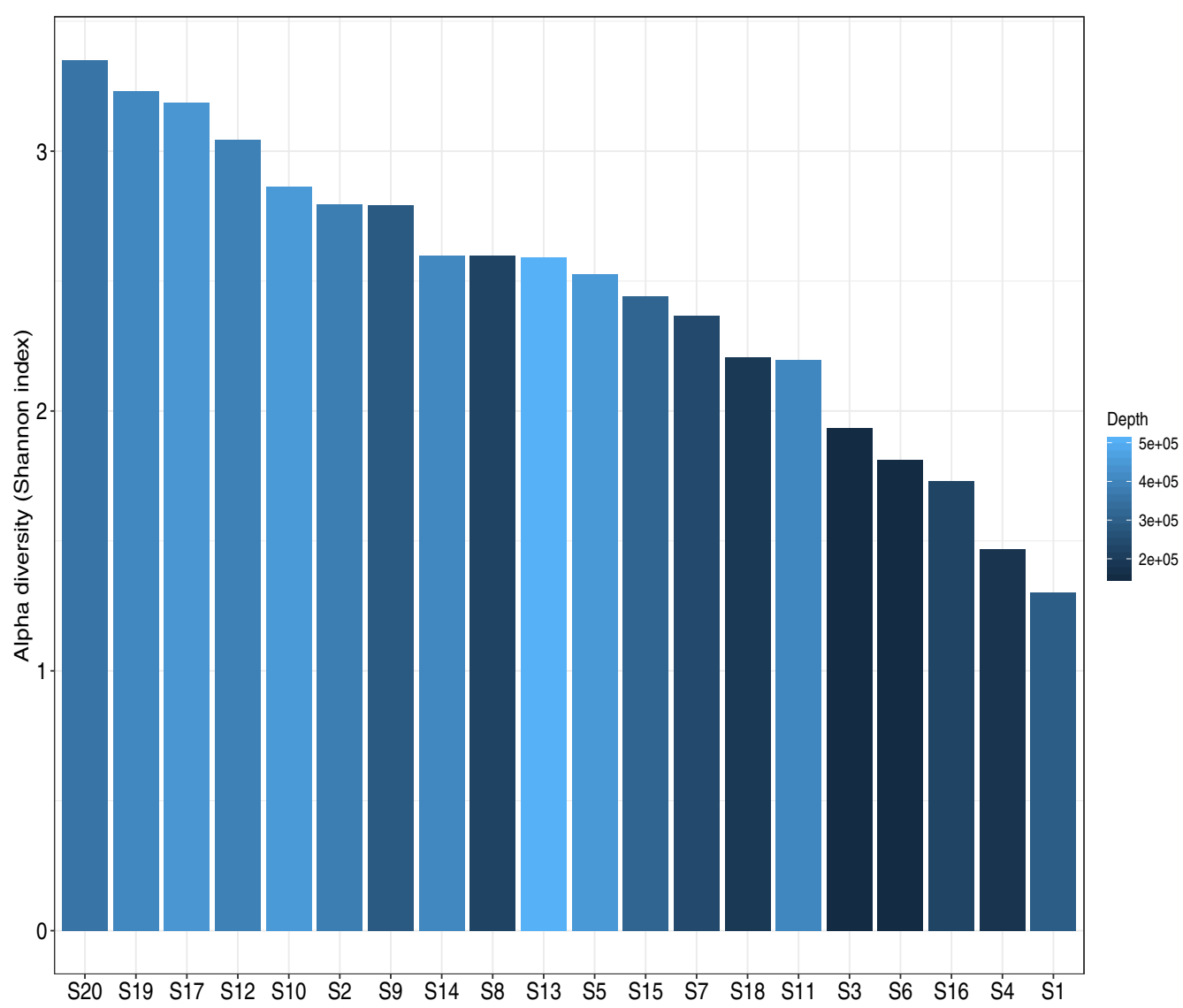

Fig. 8 a-Diversity of twenty diarrheal samples. The individual samples show variable richness and evenness of microbial diversity on the basis of Shannon index

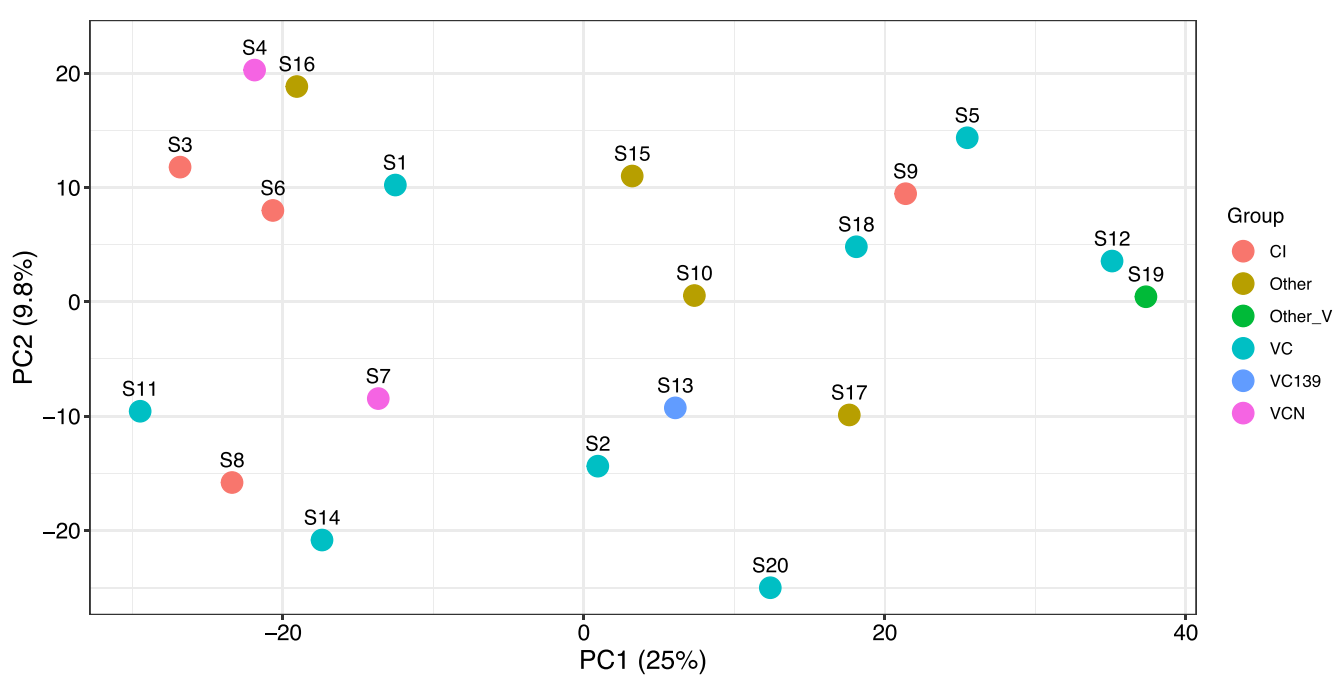

$\mathrm{Cl}=$ Co-infection; VC=V.cholerae; Other_V=other Vibrio; VCN=VC nonO1/non0139

Fig. 9 Principal component analysis of the diarrheal samples. Samples with the same diarrheal pathogen did not cluster together 


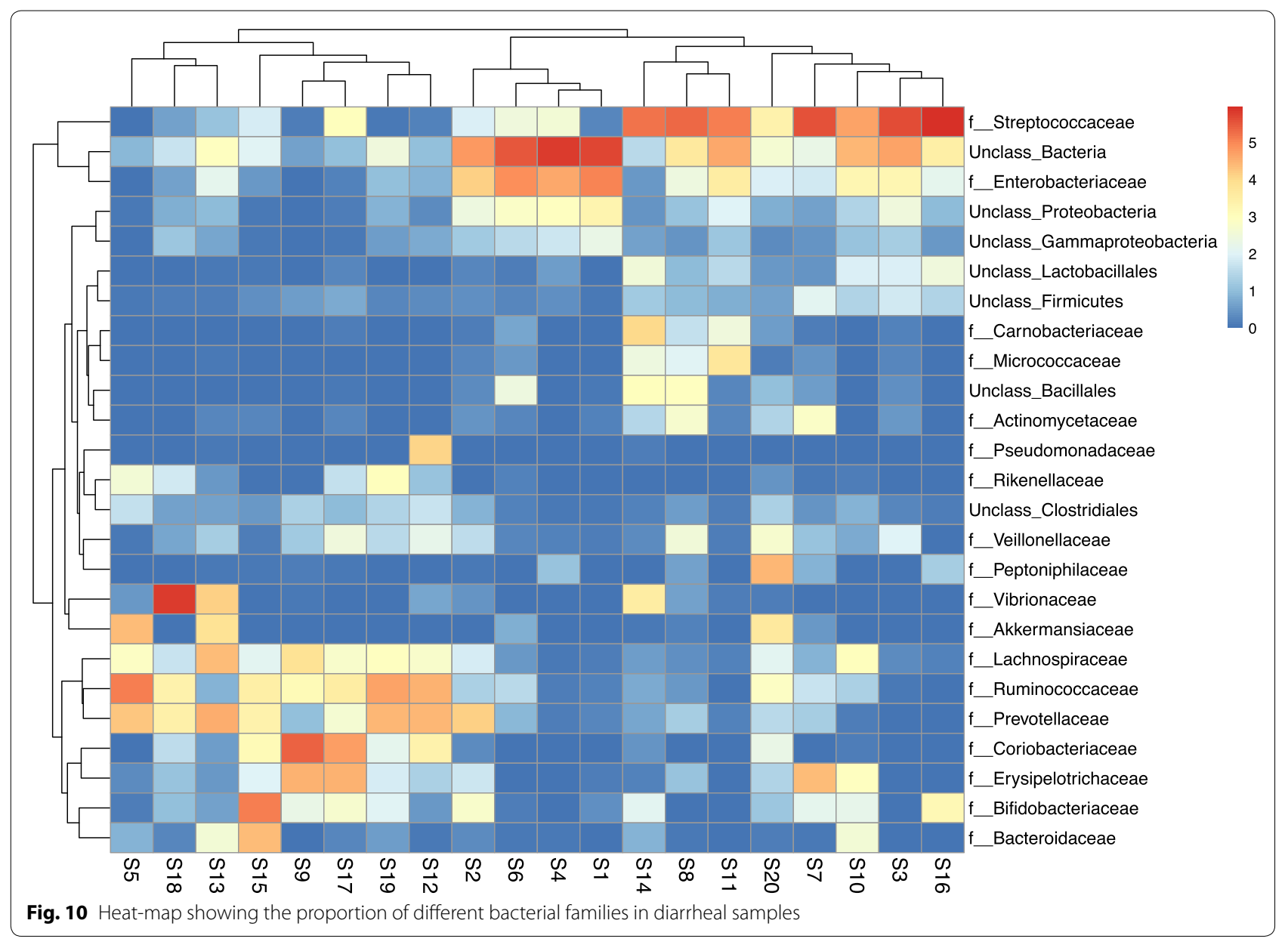

the 20 samples. It shows that most of the families occur at low abundance. The samples were found to have 18 families in common and these occurred in variable proportion even among samples associated with the same diarrheal pathogen. S1 and S20, both were associated with V.cholerae $\mathrm{O} 1$ however, in S1 family Streptococcaceae occurred in low proportion while in S20 it occurred in higher proportion while S17 associated with S. flexneri and S20 had comparable proportion of family Streptococcaceae.

\section{Resistome mapping with WGS}

WGS analysis of five samples S1, S2, S8, S9 and S10 was performed and 491 resistance determinant against the major classes of antibiotics were found by using the tool ABRicate [51]. Identities of the antimicrobial resistance genes were determined using default parameters of ABRicate, namely $75 \%$ nucleotide identity. Accordingly genetic determinants were annotated to encoding resistance against tetracycline, aminoglycosides, $\beta$-lactams, quinolone, macrolide, phenicol, glycopeptide, fosfomycin, trimethoprim, sulfonamide, lincosinamide, metronidazole, streptothricin, pleuromutilin. Resistance was high against tetracycline, $\beta$-lactams, quinolones, aminoglycosides and macrolides. Figure 11 shows all the antimicrobials against which resistance determinants were found and the relative proportion of these in each of the 5 samples. In S1 highest abundance of ARGs occurred against aminoglycosides in S2 against tetracycline, both less than 25\%, in S8 against tetracycline and it was found to be more than $60 \%$. ARGs against other classes in S8 were found to be within 5\%. In S9 highest abundance of resistance determinants occurred against tetracycline at greater than $30 \%$. In S10 equal abundance of greater than 20\% resistance determinants was found for tetracycline and quinolone. Among 5 samples, tetracycline resistance was found to be the highest in 4 samples. Therefore, $80 \%$ samples (based on calculations using 5 metagenomes) could be predicted to carry tetracycline resistance determinants. All of the samples showed resistance determinants against tetracycline, $\beta$-lactams, macrolide, aminoglycoside, phenicol and sulphonamide. Biosynthetic gene clusters (BGCs) associated with secondary metabolites involved in antimicrobial resistance were also recovered and annotated with the 

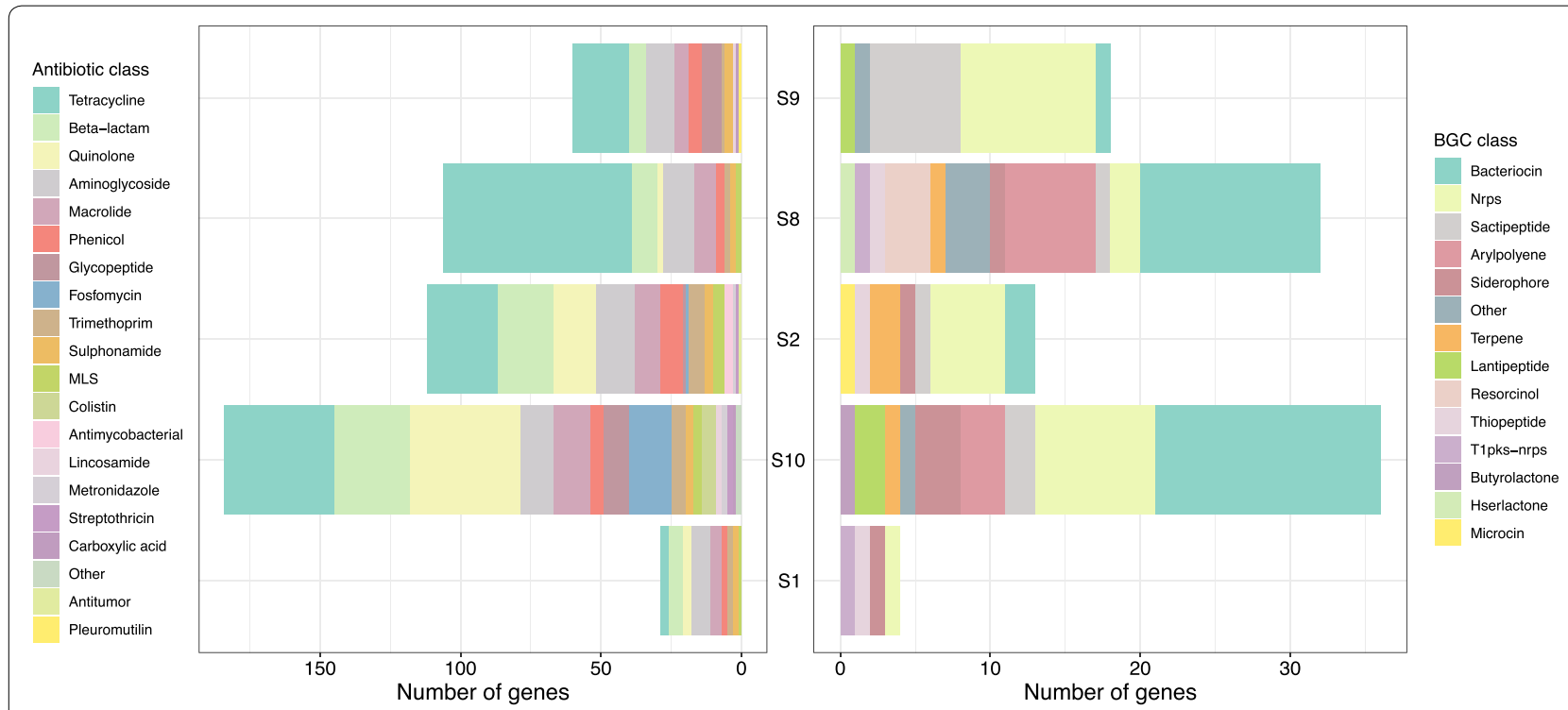

Fig. 11 Resistome of diarrheal samples. Whole genome shot-gun sequencing was used to study the resistome in five diarrheal samples. The histogram presents the relative abundance of antimicrobial resistance determinants and secondary metabolites predicted to be present in the gut microbiome of diarrheal subjects in the study

help of antiSMASH algorithm (Fig. 11). Highest number of genes were annotated to bacteriocin in S8 and S10. S8 showed the highest diversity of BCGs as 11 BCGs could be assembled followed by 9 in S10. Nonribosomal peptide synthetase (NRPS) was the only BCG that was present in all the five samples.

Genomes recovered from the 5 fecal samples were aligned using metaWRAP. It revealed the different bacterial species present in each fecal sample. Phylogenetic tree in Fig. 12 shows the different OTUs predicted to be the source of the antimicrobial resistance genes in each sample and the clonal relationship among these OTUs. The highest number of OTUs occurred in S9. Origin of ARGs in S10 could be traced to Klebsiella pneumoniae, Bacteroides B vulgatus, Bifidobacterium sp., Eggerthella lenta, Collinsella sp. and CAG-83 sp., Catenibacterium sp., Holdemanella sp., Enterococcus B faecium, Streptococcus infantarius and Streptococcus pasteurianus. Signature of Eschericia coli D occurred at highest percentage in S1 (55.84\%), S2 (50.07\%) and S8 (32.19\%) and Streptococcus infantarius signature occurred at $15.3 \%$ in S10. Figure 13 shows the 41 MAGs and their contribution towards AMR in each sample. E.coli is the highest contributor being the major MAG detected in 3 of the 5 samples. MGYG-HGUT-2778 was the major contributor in S9 while Streptococcus pasteurianus was the highest contributor in S10. In S1, the ARGs originated from Eschericia sp.

\section{Discussion}

16S rRNA amplicon sequencing was used to study the gut microbiota associated with diarrhea in twenty diarrheal samples collected from two hospitals in Kolkata to define the core and variable microbiota in this part of India. Bacterial taxonomic identification was performed by matching DNA sequence homology of the metagenomic reads generated from 20 diarrheal samples to 1,45906 reference genomes available on the Genome Taxonomy Database [50]. We have been able to identify taxonomic units at different taxonomic levels namely, phyla, class, order, family, genera and species which were found in all the twenty diarrheal samples. Therefore, it may be inferred that these constituents may be present as part of the core microbiome in diarrheal patients. However, we cannot assert to what extent the proportion of these constituents has been altered or undergone dysbiosis compared to the normal or non-diarrheal microbiota, since, comparison of diarrheal and non-diarrheal stool samples was beyond the scope of our work. Next-generation sequencing is not easily accessible due to the constraints of expenses incurred in the sequencing and analysis process [55]. Therefore, we conducted a pilot study with a small sample size of 20 diarrheal fecal samples to determine microbiota composition during diarrhea and to define a bacterial signature for diarrhea, irrespective of the pathogen causing diarrhea.

We aimed to see differences in microbiota structure based on the diarrheal pathogen that was isolated by classical microbiological method. We found in diarrheal 


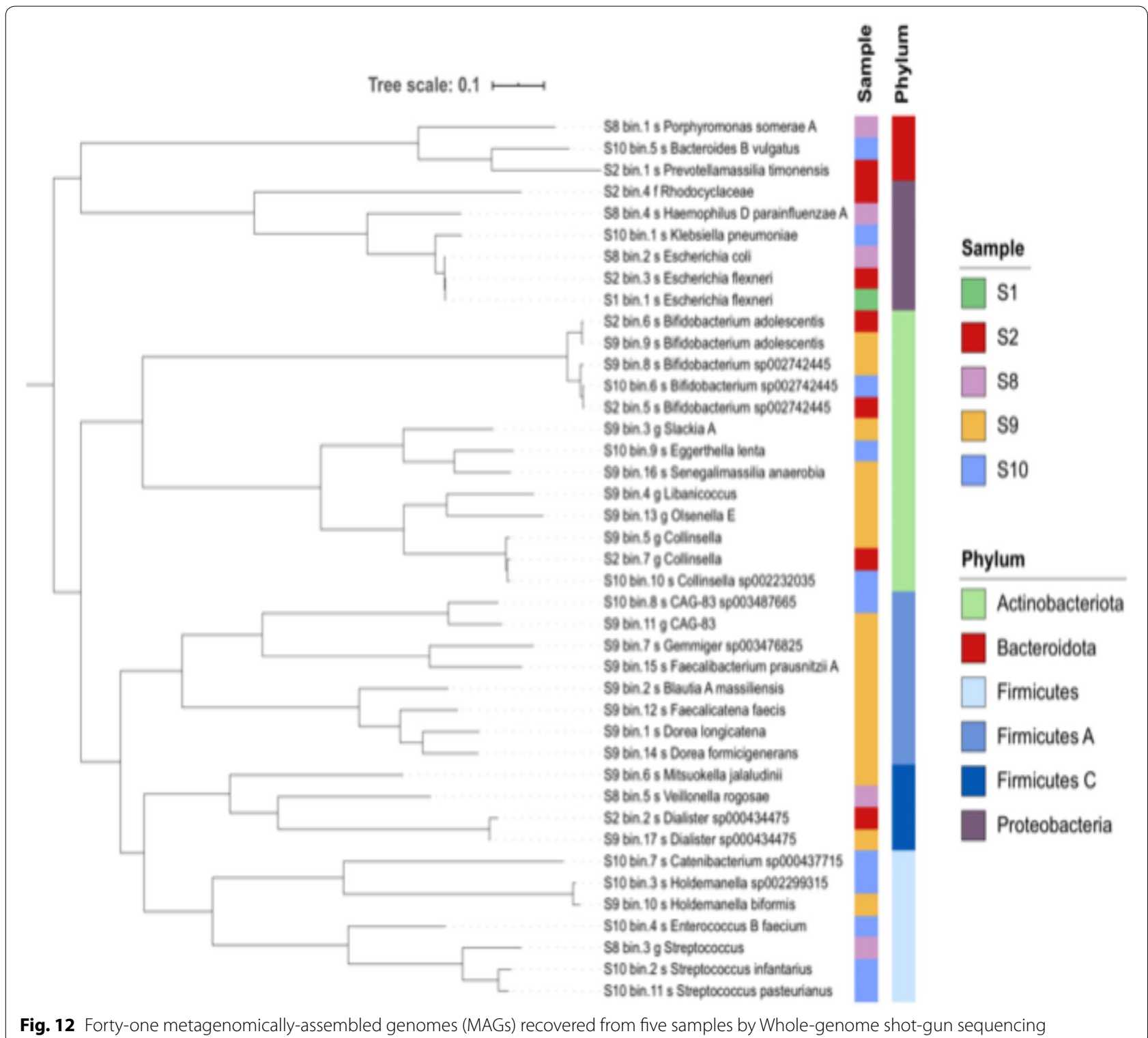

samples the dominant phylum is Firmicutes. In 11 out of 20 samples phylum Firmicutes was the most abundant phylum (Fig. 4). The Bacteroidetes/Firmicutes is an important indicator of bacterial dysbiosis [25]. The healthy gut has been found to have higher proportion of Bacteroidetes than Firmicutes [25]. 18 out of 20 diarrheal fecal samples showed higher abundance of Firmicutes than Bacteroidetes. From our study we conclude that the diarrheal gut has a higher abundance of Firmicutes than Bacteroidetes. Two samples S13 associated with V.cholerae $\mathrm{O} 139$ infection and S15 associated with Aeromonas sp. were found to have a higher proportion of Bacteroidetes compared to Firmicutes. In sample S13, which was obtained from an adult male of 40 years the dominant phylum was Bacteroidetes and in S15 obtained from a male child of 1 year old, Actinobacteria was the most abundant phylum. However, in this sample also Firmicutes was in higher abundance than Bacteroidetes. The gut microbiota primarily comprise Firmicutes, Bacteroidetes, Actinobacteria, Proteobacteria, Tenericutes and Fusobacteria [56]. The adult Microbiota is dominated by phyla Firmicutes, Bacteroidetes and Actinobacteria and depends on a host of intrinsic and extrinsic factors while the infant gut is usually dominated by phylum Actinobacteria [20, 56-58]. In sample S16, which came from a female infant of 8 months old and Aeromonas sp. was the etiologic agent as confirmed by culture method, the most dominant phylum was found to be Firmicutes. 


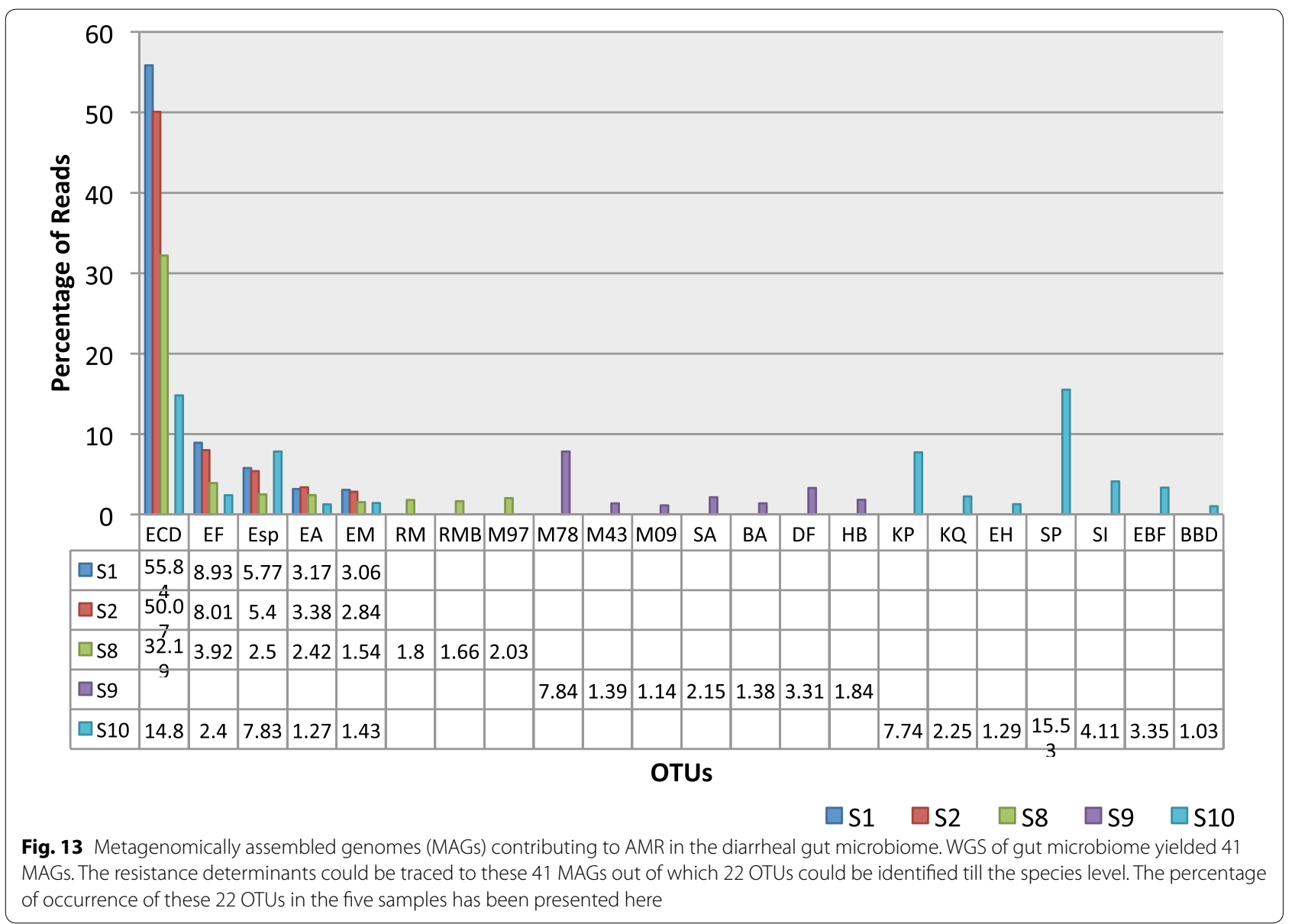

Proteobacteria was the most abundant phylum in six samples. From all these samples V.cholerae was isolated by classical culture method (Table 1, Fig. 4). B/F ratio of diarrheal patients associated with V.cholerae infection was found to be statistically significant on the basis of one sample $t$ and Wilcoxon test. This provided us with an insight into the $\mathrm{B} / \mathrm{F}$ ratio that might be associated with cholera. The study provided us with a trend in microbiota structural composition in the diarrheal gut that could also be indicative of dysbiosis. However, comparison of the profile with that of non-diarrheal subjects will help in establishing the baseline of Bacteroidetes/Firmicutes ratio. This could be assertively used as an indicator for diarrhea.

Predominance of Proteobacteria and Firmicutes are indicators of a disturbed gut microflora [55]. 42 other phyla including Tenericutes, Fusobacteria and Candidate phylum radiation (CPR) were found in some of the samples. Their proportion was found to be very minute. Although Tenericutes and Fusobacteria have been shown to be a part of the core Microbiota [56], in our study these were absent in samples like S16, which was associated with Aeromonas sp. infection. Under the superphylum PVC [59] all phyla except Omnitrophica occurred in one or the other sample. These were Planctomycetes, Verrucomicrobiae, Chlamydiae and Lentisphaerae. Verrucomicrobiae associated with primarily beneficial bacteria and of environmental origin [59] was found to be in low abundance in most of the samples in which it occurred. Core Microbiota varies with geographic location, nationality and diet among other factors [20,60]. Previous reports by other researchers in other parts of the world have shown the presence of Actinobacteria and Verrucomicrobia as dominant phyla in healthy subjects $[20,60]$. A suppression in the proportion of these phyla in the diarrheal subjects in our study indicate either a characteristic of the Indian gut microbiota or dysbiosis associated with diarrhea. A study by Das et al. showed the healthy Indian gut consistently harbours 62\% Firmicutes, 24\% Bacteroidetes, 5.2\% Actinobacteria and 4.2\% Proteobacteria and a low abundance of Verrucomicrobia, Tenericutes and Fusobacteria were found in most of the individuals participating in the study [61]. In the present study we found 38\% Firmicutes, 10\% Bacteroidetes, 12\% Actinobacteria and 
19\% Proteobacteria. The difference in the abundance of these phyla as observed in the present study could be due to diarrhea and diet, ethnicity, geographical location and other environmental factors influencing the proportion of these constituents. A study conducted by Monira et al. addressing the gut microbiota composition in healthy and malnourished children in Bangladesh showed that in healthy children Proteobacteria and Bacteroidetes accounted for $5 \%$ and $44 \%$ respectively [62]. As Eastern India and Bangladesh are comparable demographies we may assume that the lower abundance of Proteobacteria in the healthy gut observed in Bangladeshi children has been altered in diarrheal subjects resulting in higher abundance of Proteobacteria in the diarrheal subjects of the present study.

Candidate Phyla Radiation (CPR) like Candidatus Falkowbacteria, Candidatus Moranbacteria and others, belonging to the Parcubacteria group (Table 2) were found in our study. These are uncultured bacteria of environmental origin and involved in important ecological activity like sulfur-reduction and other biogeochemical cycles like carbon and hydrogen cycles [63, 64]. These are of ancient lineage, mostly symbionts or episymbionts, lack biosynthetic pathways and have not been cultured due to their stringent metabolism [64]. Our metagenomic data showed the presence of Candidatus Saccharibacteria or TM7 phylum which has been found to be a potential pathogen with a parasitic lifestyle, associated with human inflammatory mucosal diseases and often recovered from wastewater and clinical environments $[65,66]$.

The uncultivated Candidate phyla is referred to as "microbial dark matter" [66]. Its presence in diarrheal samples from patients in and around Kolkata is a matter of concern about environmental pollution and intestinal colonization of organisms with pathogenic potential. It will be interesting to investigate how they have adapted to the intestinal habitat and about the transmission of these organisms into the host from the environment. In the future it will be interesting to look for these metagenomes in healthy/non diarrheal microbiome.

In the recent years a large number of published reports attempting to define the core gut microbiome of Indians are available $[61,67]$. Bacterial composition at the genus level has been found to be influenced by location and diet [61]. Kulkarni et al. showed the presence of Prevotella sp., Bacteroides sp., Megasphaera sp., Roseburia sp., from fecal samples of 43 Indians. Das et al., showed the presence of a core microbiota comprising 54 genera from fecal samples of individuals from rural, urban and high-altitude dwellers in India [61]. Another study conducted by Lin et al. showed that healthy Bangladeshi chidren harboured more of Prevotella, Butyrivibrio, and Oscillospira and were depleted in Bacteroides [68]. Our study is the first attempt to present a core microbiota signature in diarrheal subjects from Eastern India. We found 18 genera that were present in all the 20 samples (Table 3). Prevotella sp. was absent in S10. The diarrheal etiology of this sample could not be successfully determined by culture method. This sample was from a 55 year old male from Kolkata who was hospitalized for 1 day at ID Hospital in Kolkata. Prevotella sp. has been found to be associated with the core human gut microbiome [61]. It is a pathobiont of clinical significance. A positive correlation between the upsurge of Prevotella copri and diarrhea has been estimated by previous studies [69].

The study showed the presence of commensals, pathobionts and pathogenic bacteria in the diarrheal gut microbiome. Pathobionts may cause inflammatory disorders or may cause infections in the event of compromised immunity $[70,71]$. The presence of pathogens like $V$. cholerae, Helicobacter pylori etc. in addition to the etiologic agent isolated by culture was found in many samples. This is a matter of grave concern as asymptomatic carriers act as reservoirs of infections and expedite the transmission of infections. Commensals like Bifidobacterium sp., Ruminococcus sp., Fecalibacterium sp., Lactobacillus sp., Lactococcus sp., were found in the present study and are intrinsic colonizers of the human gut [72]. Commensals play a protective role by mediating colonization resistance and preventing colonization by pathogens and opportunistic pathogens, prevent intestinal barrier impairment and suppresses pro-inflammatory factors thereby preventing diarrhea [73, 74]. Earlier studies showed that abundance of certain commensals remained unchanged before, during and after recovery from acute diarrhea in children while others like Eubacterium sp., Fecalibacterium sp., Prevotella sp., Bacteroides sp., showed marked differences during acute diarrhea and after recovery [75]. It will be interesting to investigate whether the reduction in proportion of commensals prior to diarrheal onset lay the ground for diarrheal pathogenesis. Previous studies on diarrhea associated microbiota had found a positive correlation between diarrhea and pathogenic bacteria like Eschericia sp., Shigella sp., Granulicatella sp, Streptococcus sp. [76], We found the existence of these pathogenic genera in our study subjects. Eschericia sp. was not found in S5, S7, S9, S12, S14, S18 and all of these samples were associated with $V$. cholerae infection. These findings led us to test if there is a correlation in the relative abundance of various families of pathogens and among pathogens and commensals which could be of significance for diarrheal etiology or bear implications for diarrheal treatment.

We found an association among the relative abundance of families of commensals and pathogens (Fig. 6a). Although some of the associations were not 
statistically significant it succeeded to present a trend which may be useful for understanding the agonistic and antagonistic relationship among these families and could show direction in preventive and therapeutic modules of diarrheal diseases. These correlation could become statistically significant if performed on a larger sample size. We found that the commensals Bifidobacteriaceae and Lachnospiraceae were negatively correlated with pathogens Enterobacteriaceae and Vibrionaceae. Among the pathogenic groups, family Enterobacteriaceae was higher than both Vibrionaceae and Aeromonadaceae thereby shedding light on the trend observed in gut microbiota during diarrhea. Streptococcaceae and Enterobacteriaceae were positively correlated indicating that these two pathogenic groups show the same trend in gut microbiota structural composition in diarrhea. Enterobacteriaceae are a family of potential pathogens and our study showed that these outnumber other families of potential pathogens like Vibrionaceae and Aeromonadaceae in diarrhea implying the obvious trend in diarrheal dysbiosis.

We observed differences in relative abundance among various families of bacteria in samples found to be associated with $V$. cholerae or Aeromonas sp. as etiologic agents. These are two common diarrheal pathogens and we wanted to examine if we could derive any significant association of any pathogenic or commensal family with these specific diarrheal etiology. We noted a trend in the difference in abundance of Vibrionaceae with Bifdobacteriaceae and Lachnospiraceae and Enterobacteriaceae. Vibrionaceae was higher than the commensals while lower than Enterobacteriaceae. Aeromonadaceae abundance was lower than those of the commensals and Enterobacteriaceae but the difference was non-significant. These findings suggest that in diarrhea commensals are suppressed by pathogens belonging to these families and could bear implications for probiotic therapy in diarrhea with commensal gut pathogens. This is also suggestive of the pattern of dysbiosis occurring in diarrhea. The same comparative analysis if performed in a healthy study cohort may help to determine if the observed differences in our analysis is due to dysbiosis associated with diarrhea.

Mean abundance of Enterobacteriaceae was significantly higher than that of Aeromonadaceae in the diarrheal study cohort. Significant difference in mean abundance among Bacteroidaceae, Enterobacteriaceae and Vibrionaceae was observed. These findings suggest that in diarrhea certain families of pathogens overpower others and this may lead to co-infections, co-morbidities leading to complications in diarrheal treatment.

Statistically significant positive correlation was observed among the families of commensals like Bifidobacteriaceae, Lachnospiraceae and Ruminococcaceae, indicating agonistic relationship among these and significant negative correlation among families of commensals and pathogens like Enterobacteriaceae and Lachnospiraceae and Enterobacteriaceae and Ruminococcaceae were observed. All these observations indicate antagonistic relationship bearing promise of future exploitation of these tendencies for development of probiotics.

The samples had a variable range of $\alpha$-diversity. S1 had the least while S20 had the maximum diversity. Samples like S1 and S20 associated with the same diarrheal etiologic agent, VC, had stark differences in Shannon-indices indicating that other parameters are crucial for microbiota structural composition. For analysis of $\beta$-diversity samples were grouped according to diarrheal agent isolated from it by culture method. The samples did not group into clusters based on the etiologic agent. We anticipate this was due to the small sample size and also factors other than the etiologic agent of diarrhea determining the bacterial composition in the gut.

The gut of diarrheal patients carries a high abundance of antimicrobial resistance genes (ARGs) and the members of the microbiota have been found to carry these genes in their genomes and act as reservoirs of AMR in the gut $[55,77]$. We used WGS to sequence five diarrheal samples to study the resistome and understand the origin of ARGs in the gut microbiome. We selected the fecal samples to see if variation in these aspects existed based on demography, etiology and $\alpha$-diversity. In spite of the differences in demography, etiology and $\alpha$-diversity all the samples showed the presence of the four classes of ARGS namely, tetracyclines, $\beta$-lactams, aminoglycosides and macrolides. Even though samples like S1 and S2 were associated with the same diarrheal etiology $V$. cholerae and were from the same district, 24 Parganas, their resistome analysis revealed difference in relative abundance of the same ARGs like tetracyclines, quinolones, $\beta$-lactams, aminoglycosides and macrolides. Although S1 had the lowest $\alpha$-diversity, it did not have the lowest diversity of ARGs although had the lowest number of total ARGs compared to the others. S9 and S10 were both from Kolkata but S10 had the highest number of ARGs while S9 had much lower number of ARGs and S10 had much higher relative abundance of each class of ARGs compared to S9. Moreover, quinolones were absent in S9. We conclude that in this region Eschericia sp. is the major contributor of ARGs in the gut. This is of grave concern. Eschericia sp. includes both commensals and pathogens. They are involved in metabolism and defense mechanisms [78]. Eschericia sp. are resident microbes of the gut. These will act as reservoirs for dissemination of ARGs into other bacteria in close proximity. Moreover, from the five fecal samples genomes of $E$. coli $D, E$. marmotae, E. albertii, E. fergusonii were reconstructed in 
addition to others (Fig. 13). Many of these pathogens are MDR as confirmed by previous studies [79].

We found a high abundance of resistance against tetracyclines, macrolides, aminoglycosides, quinolones and $\beta$-lactams. This presents a menacing picture of the AMR crisis in countries like India. These are last resort drugs against enteric pathogens like E.coli, K. pneumoniae, $V$. cholerae which are common diarrheal pathogens in India. Our study revealed that resistance determinants against the most important classes of antimicrobials are present in the gut of people residing in this region. This will contribute to transmission and spread to the community and the environment and lead to the emergence of MDR and XDR (Extensively Drug Resistant) strains.

Diarrhea is associated with dysbiosis of microbiota [75]. The dynamics of gut microbiota has been wellstudied in case of invading pathogens like V.cholerae [80]. We used NGS to study the gut microbiota in acute diarrheal patients in the present study. The results showed that a core microbiota exists in diarrheal patients. Specific signature of microbiota composition corresponding to distinct diarrheal etiology could not be established. We anticipate it is due to the small sample size. The trend that we observed can be confirmed by expanding the sample size in the future. The study helped to reveal the critically high abundance of AMR determinants against the most crucial drugs administered for diarrheal treatment and confirmed the existence of these determinants in the gut of diarrheal patients and originating from genomes of pathogens residing in the gut. From these ecological cross-talk future threat of infections by MDR bacteria would emanate. The study highlights the presence of asymptomatic carriers of pathogens who are serving as reservoirs of important infectious agents and expediting community transmission of diarrheal pathogens.

In the study two NGS techniques were used simultaneously. 16S rRNA amplicon sequencing helps to identify bacterial taxa but not function. WGS provides comprehensive information about both the structure and function of the microbiota. It also helps to identify the genomes contributing to those functions. Therefore we conclude that if molecular epidemiological laboratories can overcome financial constraints, WGS would be the preferred technique for investigating the constituent genomes of the microbiome and annotate their functional role.

\section{Conclusion}

The pilot study revealed significant antagonistic correlation of families of commensals like Lachnospiraceae and Ruminococcaceae with pathogens like
Enterobacteriaceae, on the basis of Spearman's correlation coefficient test. Bacteria with probiotic capability can be identified and these can be developed as probitocs for alternative therapy to replace or supplement antibiotic therapy in diarrhea. The existence of "microbial dark matter" in diarrheal gut evident from our study is indicative of contamination of the gut microbiota with rare and dangerous bacteria. This would help in epidemiological analysis to trace the origin and understand the route of transmission of members of Candidate phyla into the diarrheal gut microbiome. Consequently, it will be useful to reduce the occurrence of such organisms in the environment and the gut. Overall, the study on metagenomic sequencing of diarrheal microbiome is the first of its kind, from Eastern India revealing the core and variable microbiota associated with diarrhea and has immense implications for understanding diarrheal etiology.

\section{Methods \\ Collection of fecal samples}

Twenty diarrheal stool samples S1-S20 were collected at the IDH amd BCH, Kolkata. The donors of the fecal samples were patients suffering from acute diarrhea. They were passing liquid stool more than three times a day and were suffering from dehydration. Five of these (S1, S2, S4, S16, S17) were collected from day patients at the outpatient ward at $\mathrm{BCH}$ and the remaining fifteen were from patients admitted to the IDH for 1-3 days for receiving treatment for diarrhea. The samples were from both male and female patients of age 8 months to 56 years. Nineteen of the donors were from Kolkata and the adjacent districts in West Bengal in Eastern India while one was from the adjacent state of Bihar. The samples were brought to the Bacteriology laboratory at the adjoining National Institute of Cholera and Enteric Diseases (NICED) within few hours of collection. The samples were assigned laboratory identification code and immediately aliquoted into sterile $2 \mathrm{ml}$ cryovials (catalogue number SCT-200-SS-C$\mathrm{S}$, Corning, USA) and stored at $-80{ }^{\circ} \mathrm{C}$ for isolation of microbial DNA. A part of the sample was used for routine diagnosis of the diarrheal pathogen by culture method. A list of the samples and their demographic details are shown in Table 1 . The samples were randomly selected and were not subject to any selective bias regarding any demographic and clinical parameter. Figure 1 shows the location of West Bengal on the map of India and the state of West Bengal with its districts.

\section{Isolation of microbial DNA}

Microbial DNA was extracted by the Guanidinium thiocyanate (GITC) method according to the THSTI protocol 
described by Bag et al. [35] with minor modification. This method employs a combination of enzymatic, chemical and mechanical lysis for the complete breakdown of the bacterial cell wall, cell membrane and removal of nucleases. Accordingly, $200 \mu \mathrm{l}$ stool sample was resuspended in Tris-EDTA buffer ( $\mathrm{pH} 8.0)$ and homogenized using sterile glass beads $(2.5 \mathrm{~mm})$ and the clear suspension collected after centrifugation was subject to enzymatic lysis at $37^{\circ} \mathrm{C}$ for $1 \mathrm{~h}$ by a mixture of bacterial cell-wall lysis enzymes containing lysozyme $(10 \mathrm{mg} / \mathrm{ml})$ (catalogue number L6876, Merck, Germany), lysostaphin $(4 \mathrm{KU} / \mathrm{ml})$ (catalogue number L7386, Merck, Germany) and mutanolysin $(25 \mathrm{KU} / \mathrm{ml})$ (catalogue number M9901, Merck, Germany). $250 \mu \mathrm{l}$ of $4 \mathrm{M}$ GITCwas added to the suspension followed by $300 \mu \mathrm{l}$ of $10 \% \mathrm{~N}$-Lauryl sarcosine and incubated at $37{ }^{\circ} \mathrm{C}$ for $10 \mathrm{~min}$. Mechanical disruption by $0.1 \mathrm{~mm}$ zirconia beads (BioSpec Products Inc., USA) ensued in a mini beadbeater (catalogue number 607EUR, BioSpec Products Inc., USA) using a 2 min cycle comprising $30 \mathrm{~s}$ beating and $30 \mathrm{~s}$ rest and followed by washing in PolyVinylPolyPyrollidone (PVPP) (catalogue number 77627, Merck, Germany). Removal of RNA was done using RNase A $(10 \mathrm{mg} / \mathrm{ml})$ (catalogue number R6513, Merck, Germany) and incubating the suspension for $30 \mathrm{~min}$ at $37^{\circ} \mathrm{C}$. DNA was finally extracted by adding $96 \%$ chilled ethanol and spinning at $14,000 \mathrm{rpm}$ for $10 \mathrm{~min}$ at $4{ }^{\circ} \mathrm{C}$. The pellet was air-dried followed by estimation of DNA concentration with NanoDrop spectrophotometer and Qubit ${ }^{\circledR}$ dsDNA HS Assay Kit (catalogue number Q32854, Invitrogen, USA). The DNA concentration was in the optimal range and estimated at $1 \mathrm{ng} / \mu \mathrm{l}-400 \mathrm{ng} / \mu \mathrm{l}$. The 20 DNA samples were used for library preparation for $16 \mathrm{~S} \mathrm{V3-V4} \mathrm{ampli-}$ con sequencing and 5 of the 20 DNA samples were used for WGS sequencing for resistome analysis.

\section{$16 S$ rDNA sequencing and metagenomic analysis}

$16 \mathrm{~S}$ V3-V4 metagenome libraries were prepared using region-specific primers. DNA samples were loaded on gel to examine the bands followed by $0.7 \times$ Hiprep bead clean up using HighPrep ${ }^{\text {TM }}$ clean up system (catalogue numberAC-60050, MagBio genomics Inc., USA) to avoid impurities and amplified for 26 cycles of round 1 PCR using KAPA HiFi Hot-Start PCR Kit (catalogue number KM2602, KAPA Biosystems Inc., Boston, MA, USA). The forward and reverse primer concentration was kept at 5 $\mu \mathrm{M}$ each. The amplicons were analyzed on $1.2 \%$ agarose gel. $1 \mu \mathrm{l}$ of diluted round 1 PCR amplicons were used for Indexing PCR (Round 2). Round 1 PCR amplicons were amplified for 10 cycles to add Illumina sequencing barcoded adaptors (Nextera XT v2 Index Kit, catalogue number FC-131-1002 Illumina Inc., CA, USA). Illumina Adapter Sequences used were: 5'-AATGATACGGCG ACCACCGAGATCTACAC[i5]TCGTCGGCAGCGTC and 5'-CAAGCAGAAGACGGCATACGAGAT[i7] GTC TCGTGGGCTCGG where [i5, i7] are unique dual index sequences to identify sample-specific sequencing data.

Round 2 PCR amplicons (sequencing libraries) were analyzed on 1.2 percent agarose gel, cleaned using HighPrep $^{\text {TM }}$ clean up system and quality checked. The library was diluted to $4 \mathrm{nM}$ using $10 \mathrm{mM}$ Tris $(\mathrm{pH} 8.5)$ and $5 \mu \mathrm{l}$ of each library was aliquotted and mixed to pool the libraries. The pooled library was denatured by addition of $\mathrm{NaOH}$ followed by heat denaturation and the DNA samples were diluted and finally loaded onto the Illumina MiSeq system and sequencing was performed to generate $(300 * 2) \mathrm{V} 3-\mathrm{V} 4$ paired-end reads.

The Illumina paired end V3-V4 raw reads (300*2) were submitted to the European Nucleotide Archive (ENA) for validation and further analysis using the MGnify pipeline provided by the EMBL server. The study was assigned the number MGYS00005131. The raw reads were processed using MGnify v4.1. SeqPrep [36] was used to merge the overlapping raw reads into a single longer read. Trimmomatic [37] and Biopython [38] were used to trim and filter these initial reads by removing $>10 \%$ undetermined nucleotides and adapter sequences and filtering out $<100 \mathrm{bp}$ long sequences to generate processed reads which were annotated using MAPseq [39] framework for taxonomic classification and Operational Taxonomic Unit (OTU) mapping. For classification of OTUs, pairedend reads with $>97 \%$ sequence similarity were considered. The sequences of raw and processed reads can be accessed through the EMBL server with the accession number MGYS00005131.For multivariate analysis and graphical representation of the metadata tools Codaseq [40], Vegan [41] and Ape [42] on the Phyloseq [42] package, ggplot2 [43] on R Studio (R studio Inc, Boston, MA, USA) were used. Biom files generated by MAPseq in the MGnify pipeline were imported into $\mathrm{R}$ package. Principal component Analysis (PCA) was performed using the Phyloseq package for analysis of abundance of OTUs and entitities within different taxonomic ranks namely, phylum, class, order, family, genera and species. Abundance was expressed as percentage. Relative abundance of different entities within a taxonomic level was represented as histogram to show taxonomic diversity and abundance. $0-5$ percent was used as the threshold. The top fifteen to twenty-five OTUs within each taxon were plotted for each sample. $\alpha$-diversity was calculated to estimate species richness and evenness of each sample. Accordingly, OTUs were rarefied at even depth and Shannon index was calculated. To calculate $\beta$-diversity between the samples ordination was performed and principal coordinates plots were generated based on pairwise weighted UniFrac distances. Pie, bar, stacked and interactive krona charts were generated by the taxonomic analysis steps of the MGnify 
v 4.1 pipeline. Bacteroidetes/Firmicutes ratio was calculated and compared among the 20 samples. For bivariate analysis normal distribution, $\mathrm{z}$-score, unpaired $\mathrm{t}$-tests, ANOVA (Analysis of Variance) and one sample $t$ and Wilcoxon tests were calculated to represent the statistical significance of the taxonomic composition and abundance data. Correlation coefficient using Spearman's rank coefficient test was used to study correlation among abundance of families Bifidobacteriaceae, Lachnospiraceae, Ruminococcaceae, Enterobacteriaceae, Vibrionaceae, Streptococcaceae to derive if any significant association existed among them in diarrhea. Kruskal-wallis test was used to compare abundance of three families of commensal bacteria namely Bifidobacteriaceea, Lachnospiraceae and Ruminococcaceae and three families of pathogenic bacteria namely Enterobacteriaceae, Bacteroidaceae and Vibrionaceae to see if an association could be established among the relative abundance of these families which could have a significance for diarrheal etiology.

Unpaired t-test was used to compare difference in relative abundance of Bifidobacteriaceae with Enterobacteriaceae and Vibrionaceae, Lachnospiraceae with Enterobacteriaceae and Vibrionaceae, also between Enterobacteriaceae and Vibrionaceae and between Enterobacteriaceae and Aeromonadaceae in diarrhea and calculations were based on 20 samples. Wilcoxon matched-pairs signed rank test was used to compare difference in relative abundance of Vibrionaceae with Bifdobacteriaceae, Enterobacteriaceae, Lachnospiraceae and Ruminococcaceae in samples diagnosed with Vibrio sp. by culture method. Unpaired t-test was used to compare differences in relative abundance of Aeromonadaceae with Bifidobacteriaceae, Enterobacteriaceae, Lachnospiraceae in samples diagnosed with Aeromonas sp. by culture method.

Figure 2 presents the workflow of library preparation, sequencing and metagenomic analysis.

\section{WGS sequencing and resistome analysis}

De novo sequencing of DNA from five diarrheal samples S1, S2, S8, S9 and S10 was performed for resistome profiling and to understand the presence of secondary metabolites associated with AMR present in the diarrheal metagenomes. The samples were from three different districts of West Bengal, suffering from diarrhea due to single infection or polymicrobial infections or unresolved etiology (Table 1) and with different $\alpha$-diversity. Nextera ${ }^{\circledR}$ XT Library Preparation Kit (catalogue number FC-131-1024, Illumina Inc., CA, USA) was used to prepare paired-end libraries according to the protocol documented by Illumina (Illumina Inc., CA, USA) [44]. Accordingly, $1 \mathrm{ng}$ of Qubit quantified genomic DNA was tagmented (fragmented and adaptor tagged) using
Amplicon Tagment Mix from the Nextera XT Kit. Twelve cycles of Indexing-PCR $\left(72{ }^{\circ} \mathrm{C}\right.$ for $3 \mathrm{~min}$ followed by denaturation at $95{ }^{\circ} \mathrm{C}$ for $30 \mathrm{~s}$, cycling $\left(95{ }^{\circ} \mathrm{C}\right.$ for $10 \mathrm{~s}$, $55{ }^{\circ} \mathrm{C}$ for $30 \mathrm{~s}, 72{ }^{\circ} \mathrm{C}$ for $30 \mathrm{~s}$ ) and $72{ }^{\circ} \mathrm{C}$ for $5 \mathrm{~min}$ ) were performed on the adapter tagged DNA to enrich the adapter-tagged fragments. The PCR product was purified using JetSeq Magnetic Beads (Bio, 68031). Quantification of the prepared library was performed using Qubit fluorometer according to the manufacturer's instructions. The universal adapter sequence was 5'AATGAT ACGGCGACCACCGAGATCTACACTCTTTCCCTA CACGACGCTCTTCCGATCT and adapter index was 5'GATCGGAAGAGCACA CGTCTGAACTCCAGT CAC[INDEX]ATCTCGTATGCCGTCTTCTGCTTG.

The libraries were pooled and these were sequenced in the Illumina MiSeq System (Illumina Inc., CA, USA) to generate paired-end raw reads. The raw reads were passed through the metaSPAdes $v$ 3.9.1 [45] assembler pipeline after initial quality check with FastQC [46] followed by removal of adapters and low quality bases towards 3 '-end by the program TrimGalore [47] and BWA (Burrows-Wheeler Aligner) [48] that removes host contaminants. Binning was done using the software metaWRAP [49] and taxonomic annotation and mapping was done using the GTDB Toolkit (GTDB-Tk) [50]. The contigs generated by the metaSPAdes pipeline was used for screening for acquired antimicrobial resistance genes using the tool ABRicate [51] and the program antiSMASH [52] was used for screening and annotation of secondary metabolite biosynthesis gene clusters (BGCs). Multivariate analysis and graphical representation of the metagenomic datasets were performed with ggplot2 on R Studio (R studio Inc, Boston, MA, USA).

\section{Culture of diarrheal pathogens from fecal samples}

The fecal samples S1-S20 were streaked onto selective and differential media plates for the isolation of suspected diarrheal pathogens, Vibrio sp., E. coli, Salmonella sp., Shigella sp., Aeromonas sp., Campylobacter sp. in the Bacteriology Laboratory at NICED. Accordingly, bacterial culture plates TCBS (Thiosulfate-citrate-bile salts-sucrose), HEA (Hektoen enteric agar), XLD (Xylose Lysine Deoxycholate), Mac Conkey, Blood agar were used for each fecal specimen. Culture plates were incubated overnight at $37^{\circ} \mathrm{C}(3-5$ days for Campylobacter sp.) and single colonies from the culture positive plates were used for phenotypic confirmation of diarrheal pathogens with biochemical tests [53, 54]. The confirmed strains were stored in nutrient agar. 


\section{Supplementary information}

Supplementary information accompanies this paper at https://doi. org/10.1186/s13099-020-00371-8.

Additional file 1. Hierarchical classification of taxonomic entities found from metagenomic sequencing in the study.

\section{Acknowledgements}

We are grateful to Dr. G. Balakrish Nair for his contribution to the study. We are thankful to Dr. Robert D. Finn and Dr. Alexandre Almeida at EMBL-EBI for their immense help with the analysis of the metagenomics data.

\section{Authors' contributions}

$\mathrm{RD}$ designed the study, performed the experiments, analyzed the data and wrote the manuscript; AKM did experiments and wrote the manuscript; SD planned experiments and analyzed the data. All authors read and approved the final manuscript.

\section{Funding}

We thank the Department of Health Research, Ministry of Health and Family Welfare, India, for the financial support we received for the study via grant number 12015/01/2018-HR.

\section{Availability of data and materials}

The datasets supporting the conclusions of this article are included within the article. DNA sequences have been deposited in the European Nucleotide Archive (ENA)

\section{Ethics approval and consent to participate}

The study was approved by the Institutional Ethics Committee and the Department of Health Research.

\section{Consent for publication}

Demographic details of donors of diarrheal stool were collected with their consent and all the personal information was kept confidential under lock and key.

\section{Competing interests}

The authors declare that they have no competing interests.

Received: 23 April 2020 Accepted: 23 June 2020

Published online: 07 July 2020

\section{References}

1. GBD 2016 Diarrhoeal Disease Collaborators. Estimates of the global, regional, and national morbidity, mortality, and aetiologies of diarrhoea in 195 countries: a systematic analysis for the Global Burden of Disease Study 2016. Lancet Infect Dis. 2018;18(11):1211-28.

2. https://www.who.int/news-room/fact-sheets/detail/diarrhoeal-disease Accessed on 3rd December,2019.

3. https://www.cdc.gov/healthywater/pdf/global/programs/Globaldiar rhea508c.pdf Accessed on 3 Dec 2019.

4. Guerrant RL, Schorling JB, McAuliffe JF, De Souza MA. Diarrhea as a cause and an effect of malnutrition: diarrhea prevents catch-up growth and malnutrition increases diarrhea frequency and duration. Am J Trop Med Hyg. 1992;47(1):28-35.

5. Brander RL, Pavlinac PB, Walson JL, John-Stewart GC, Weaver MR, Faruque $A S G$, et al. Determinants of linear growth faltering among children with moderate-to-severe diarrhea in the Global Enteric Multicenter Study. BMC Med. 2019;17(1):214.

6. Kotloff KL, Nasrin D, Blackwelder WC, Wu Y, Farag T, Panchalingham S, et al. The incidence, aetiology, and adverse clinical consequences of less severe diarrhoeal episodes among infants and children residing in lowincome and middle-income countries: a 12-month case-control study as a follow-on to the Global Enteric Multicenter Study (GEMS). Lancet Glob Health. 2019;7(5):e568-84.
7. Semba RD, de Pee S, Ricks MO, Sari M, Bloem MW. Diarrhea and fever as risk factors for anemia among children under age five living in urban slum areas of Indonesia. Int J Infect Dis. 2008;12(1):62-70.

8. Kamath NA, Shetty K, Unnikrishnan B, Kaushik S, Rai SN. Prevalence, patterns, and predictors of diarrhea: a spatial-temporal comprehensive evaluation in India. BMC Public Health. 2018. https://doi.org/10.1186/ s12889-018-6213-z

9. https://www.nhp.gov.in/disease/digestive/intestines/diarrhoea. Accessed on 3rd Dec 2019

10. GBD DiarrhoealDiseases Collaborators. Estimates of global, regional, and nationalmorbidity, mortality, and aetiologies of diarrhoealdiseases: a systematic analysis for the Global Burden of Disease Study 2015. Lancet Infect Dis. 2017;17(9):909-48.

11. Liu L, Chu Y, Oza S, Hogan D, Perin J, Bassani DG, Ram U, et al. National, regional, and state-level all-cause and cause-specific under-5 mortality in India in 2000-15: a systematic analysis with implications for the Sustainable Development Goals. Lancet Glob Health. 2017;7(6):e721-34.

12. Raju B, Parikh RP, Vetter W, Kolhapure S. Epidemiology of rotavirus gastroenteritis and need of high rotavirus vaccine coverage with early completion of vaccination schedule for protection against rotavirus diarrhea in India: a narrative review. Indian J Public Health. 2019;63:243-50.

13. De R. Metagenomics: aid to combat antimicrobial resistance in diarrhea. Gut Pathog. 2019. https://doi.org/10.1186/s13099-019-0331-8.

14. D'Argenio V, Salvatore F. The role of the gut microbiome in the healthy adult status. Clin Chim Acta. 2015;451:97-102.

15. Carding S, Verbeke K, Vipond DT, Corfeand BM, Owen LJ. Dysbiosis of the gut microbiota in disease. Microbial Ecol Health Dis. 2015:26:26191.

16. Turnbaugh P, Ley R, Hamady M, Fraser CM, Knight R, Gordon Jl. The human microbiome project. Nature. 2007:449:804-10.

17. Li J, Jia H, Cai X, Huanzi Z, Feng Q, Sunagawa S, et al. An integrated catalog of reference genes in the human gut microbiome. Nat Biotechnol. 2014;32:834-41.

18. King CH, Desai H, Sylvetsky AC, LoTempio J, Ayanyan S, Carrie J, et al. Baseline human gut microbiota profile in healthy people and standard reporting template. PLoS ONE. 2019;14(9):e0206484.

19. Qin J, Li R, Raes J, Arumugam M, Burgdorf KS, Manichanh C, et al. A human gut microbial gene catalogue established by metagenomic sequencing. Nature. 2010;464(7285):59-65.

20. Nishijima S, Suda W, Oshima K, Kim SW, Hirose Y, Morita H, et al. The gut microbiome of healthy Japanese and its microbial and functional uniqueness. DNA Res. 2016;23(2):125-33.

21. Milani C, Duranti S, Bottacini F, Casey E, Turroni F, Mahony J, et al. The First microbial colonizers of the human gut: composition, activities, and health implications of the infant gut microbiota. Microbiol Mol Biol Rev. 2017;81(4):e00036.

22. Dhakan DB, Maji A, Sharma AK, Saxena R, Pulikkan J, Grace T, et al. The unique composition of Indian gut microbiome, gene catalogue, and associated fecal metabolome deciphered using multi-omics approaches. GigaScience. 2019;8(3):giz004

23. Cuevas-Sierra A, Ramos-Lopez O, Riezu-Boj Jl, Milagro FI, Martinez JA. Diet, gut microbiota, and obesity: links with host genetics and epigenetics and potential applications. Advances in Nutrition. 2019;10(Suppl 1):S17-30.

24. Selber-Hnatiw S, Sultana T, Tse W, Abdollahi N, Abdullah S, Al Rahbani J, et al. Metabolic networks of the human gut Microbiota. Microbiology. 2019. https://doi.org/10.1099/mic.0.000853.

25. Youmans BP, Ajami NJ, Jiang ZD, Campbell F, Wadsworth WD, Petrosino JF, et al. Characterization of the human gut microbiome during travelers' diarrhea. Gut Microbes. 2015;6(2):110-9.

26. Maruvada P, Leone V, Kaplan LM, Chang EB. The human microbiome and obesity: moving beyond associations. Cell Host Microbe. 2017;22(5):589-99.

27. Rajagopala SV, Vashee S, Oldfield LM, Suzuki Y, Venter JC, Telenti A, et al. The human microbiome and cancer. Cancer Prev Res. 2017;10(4):226-34.

28. Barko PC, McMichael MA, Swanson KS, Williams DA. The gastrointestinal microbiome: a review. J Vet Intern Med. 2018:32(1):9-25.

29. Medina DA, Li T, Thomson P, Artacho A, Pérez-Brocal V, Moya A. Crossregional view of functional and taxonomic microbiota composition in obesity and post-obesity treatment shows country specific microbial contribution. Front Microbiol. 2019;17(10):2346. 
30. Monira S, Nakamura S, Gotoh K, Izutsu K, Watanabe H, Alam NH, et al. Metagenomic profile of gut microbiota in children during cholera and recovery. Gut Pathog. 2013;5(1):1.

31. Pereira-Marques J, Ferreira RM, Pinto-Ribeiro I, Figueiredo C. Helicobacter pylori infection, the gastric microbiome and gastric cancer. In: Kamiya S, Backert S, editors. Helicobacter pylori in human diseases advances in experimental medicine and biology. Cham: Springer; 2019.

32. Rouhani S, Griffin NW, Yori PP, Olortegui MP, Salas MS, Trigoso TR, et al. Gut microbiota features associated with Campylobacter burden and postnatal linear growth deficits in a Peruvian birth cohort. Clin Infect Dis. 2019. https://doi.org/10.1093/cid/ciz906.

33. Endt K, Stecher B, Chaffron S, Slack E, Tchitchek N, Benecke A, et al. The microbiota mediates pathogen clearance from the gut lumen after non-typhoidal Salmonella diarrhea. PLoS Pathog. 2010. https://doi. org/10.1371/journal.ppat.1001097.

34. Braun T, Di Segni A, BenShoshan M, Asaf R, Squires JE, FarageBarhom $S$, et al. Fecal microbial characterization of hospitalized patients with suspected infectious diarrhea shows significant dysbiosis. Sci Rep. 2017;7(1):1088.

35. Bag S, Saha B, Mehta O, Anbumani D, Kumar N, Dayal M, et al. An improved method for high quality metagenomics DNA extraction from human and environmental samples. Sci Rep. 2016;6:26775.

36. https://github.com/jstjohn/SeqPrep. Accessed December 2019.

37. Bolger AM, Lohse M, Usadel B. Trimmomatic: a flexible trimmer for Illumina sequence data. Bioinformatics. 2014;30(15):2114-20.

38. https://github.com/biopython/biopython. Accessed Dec 2019.

39. Rodrigues MJF, Schmidt TSB, Tackmann J, von Mering C. MAPseq: highly efficient k-mer search with confidence estimates, for rRNA sequence analysis. Bioinformatics. 2017;33(23):3808-10.

40. https://github.com/ggloor/CoDaSeq. Accessed Dec 2019.

41. https://rdrr.io/cran/vegan/. Accessed Dec 2019.

42. McMurdie PJ, Holmes S. phyloseq: an R package for reproducible interactive analysis and graphics of microbiome census data. PLOS ONE. 2013;8(4):e61217

43. https://ggplot2.tidyverse.org/reference/index.html. Accessed Dec 2019.

44. https://support.illumina.com/content/dam/illuminasupport/documents/ documentation/chemistry_documentation/samplepreps_nextera/nexte ra-xt/nextera-xt-library-prep-reference-guide-15031942-05.pdf. Accessed 19 Dec 2019

45. Nurk S, Meleshko D, Korobeynikov A, Pevzner PA. metaSPAdes: a new versatile de novo metagenomics assembler. Genom Res. 2017;27(5):824-34.

46. Andrews S. FastQC: a quality control tool for high throughput sequence data. 2010. http://www.bioinformatics.babraham.ac.uk/projects/fastqc/. Accessed Dec 2019.

47. Krueger F. Trim Galore!: A wrapper tool around Cutadapt and FastQC to consistently apply quality and adapter trimming to FastQ files.2015. http://www.bioinformatics.babraham.ac.uk/projects/trim_galore/ Accessed Dec 2019.

48. Li H, Durbin R. Fast and accurate short read alignment with BurrowsWheeler Transform. Bioinformatics. 2009;25:1754-60.

49. Uritskiy GV, DiRuggiero J, Taylor J. MetaWRAP_a flexible pipeline for genome-resolved metagenomic data analysis. Microbiome. 2018. https:// doi.org/10.1186/s40168-018-0541-1.

50. Chaumeil PA, Mussig AJ, Hugenholtz P, Parks DH. GTDB-Tk: a toolkit to classify genomes with the Genome Taxonomy Database. Bioinformatics. 2019. https://doi.org/10.1093/bioinformatics/btz848.

51. https://github.com/tseemann/abricate. Accessed December 2019.

52. https://antismash.secondarymetabolites.org/\#!/about. Accessed Dec 2019.

53. Laboratory methods for the diagnosis of epidemic dysentery and cholera. Centers for Disease Control and Prevention, Atlanta, Georgia. 1999.

54. Mac Faddin JF. Biochemical tests for identification of medical bacteria. 3rd ed. Lippincott Williams and Wilkins; 2000.

55. De R, Mukhopadhyay AK, Dutta S. Molecular analysis of selected resistance determinants in diarrheal fecal samples from Kolkata, India reveals an abundance of resistance genes and the potential role of the microbiota in its dissemination. Front Public Health. 2020. https://doi. org/10.3389/fpubh.2020.00061.

56. Turroni F, Milani C, Duranti S, Lugli GA, Bernasconi S, Margolles A, et al. The infant gut microbiome as a microbial organ influencing host wellbeing. Ital J Pediatr. 2020;46(1):16.

57. Maria D, Firmesse O, Levenez F, Guimaraes V, Sokol H, Dore J, et al. The Firmicutes/Bacteroidetes ratio of the human microbiota changes with age. BMC Microbiol. 2009. https://doi.org/10.1186/1471-2180-9-123.

58. Arumugam M, Raes J, Pelletier E, Paslier DL, Yamada T, Mende DR, et al. Enterotypes of the human gut microbiome. Nature. 2011;473(7346):174-80

59. Spring S, Bunk B, Spröer C, Schumann P, Rohde M, Tindall BJ, et al. Characterization of the first cultured representative of Verrucomicrobia subdivision 5 indicates the proposal of a novel phylum. ISME J. 2016;10(12):2801-16.

60. Fujio-Vejar S, Vasquez Y, Morales P, Magne F, Vera-Wolf $P$, Ugalde JA, et al. The gut microbiota of healthy Chilean subjects reveals a high abundance of the phylum Verrucomicrobia. Front Microbiol. 2017;8:1221.

61. Das B, Ghosh TS, Kedia S, Rampal R, Saxena S, Bag S, et al. Analysis of the gut microbiome of rural and urban healthy Indians living in sea level and high altitude areas. Sci Rep. 2018;8:10104.

62. Monira S, Nakamura S, Gotoh K, Izutsu K, Watanabe H, Alam NH, et al. Gut microbiota of healthy and malnourished children in Bangladesh. Front Microbiol. 2011;2:228.

63. Anantharaman K, Hausmann B, Jungbluth SP, Kantor RS, Lavy A, Warren $L A$, et al. Expanded diversity of microbial groups that shape the dissimilatory sulfur cycle. ISME J. 2018;12(7):1715-28.

64. Brown CT, Hug LA, Thomas BC, Sharon I, Castelle CJ, Singh A, et al. Unusual biology across a group comprising more than $15 \%$ of domain Bacteria. Nature. 2015;523(7559):208-11.

65. He X, McLean JS, Edlund A, Yooseph S, Hall AP, Liu SY, et al. Genomics and physiology of TM7. Proc Natl Acad Sci. 2015;112(1):244-9.

66. Ferrari B, Winsley T, Ji M, Neilan B. Insights into the distribution and abundance of the ubiquitous Candidatus Saccharibacteria phylum following tag pyrosequencing. Sci Rep. 2015:4:3957.

67. Kulkarni AS, Kumbhare SV, Dhotre DP, Shouche YS. Mining the core gut microbiome from a sample Indian population. Indian J Microbiol. 2019;59(1):90-5.

68. Lin A, Bik EM, Costello EK, Dethlefsen L, Haque R, Relman DA, et al. Distinct distal gut microbiome diversity and composition in healthy children from Bangladesh and the United States. PLoS ONE. 2013;8(1):e53838. https://doi.org/10.1371/journal.pone.0053838.

69. Gilchrist CA, Petri SE, Schneider BN, Daniel JR, Nona J, Sharmin B, et al. Role of the gut microbiota of children in diarrhea due to the protozoan parasite Entamoeba histolytica. J Infect Dis. 2016;213(10):1579-85. https:// doi.org/10.1093/infdis/jiv772.

70. Chow J, Tang H, Mazmanian SK. Pathobionts of the gastrointestinal microbiota and inflammatory disease. Curr Opin Immunol. 2011;23(4):473-80.

71. Wong SCY, Poon RWS, Chen JHK, Tse H, Lo JYC, Ng TK, et al. Corynebacterium kroppenstedtii is an emerging cause of mastitis especially in patients with psychiatric illness on antipsychotic medication. Open Forum Infect Dis. 2017;4(2):ofx096.

72. Thursby $\mathrm{E}$, Juge N. Introduction to the human gut microbiota. Biochem J. 2017;474(11):1823-36.

73. O'Loughlin JL, Samuelson DR, Braundmeier-Fleming AG, White BA, Haldorson GJ, Stone JB, et al. The intestinal microbiota influences Campylobacter jejuni colonization and extraintestinal dissemination in mice. Appl Environ Microbiol. 2015;81(14):4642-50.

74. Tanabe S, Suzuki T, Wasano Y, Nakajima F, Kawasaki H, Tsuda T, et al. Antiinflammatory and intestinal barrier-protective activities of commensal lactobacilli and Bifidobacteria in thoroughbreds: role of probiotics in diarrhea prevention in neonatal thoroughbreds. J Equine Sci. 2014;25(2):37-43.

75. Balamurugan R, Janardhan HP, George S, Raghava YMV, Muliyil YJ, Ramakrishna BS. Molecular studies of fecal anaerobic commensal bacteria in acute diarrhea in children. J Pediatr Gastroenterol Nutr. 2008;46:514-9. 
76. Pop M, Walker AW, Paulson J, Lindsay B, Antonio M, Hossain MA, et al. Diarrhea in young children from low-income countries leads to largescale alterations in intestinal microbiota composition. Genome Biol. 2014;15(6):R76

77. Bag S, Ghosh TS, Banerjee S, Mehta O, Verma J, Dayal M, et al. Molecular insights into antimicrobial resistance traits of commensal human gut microbiota. Microb Ecol. 2019;77:546-57.

78. Reeves PR, Liu B, Zhou Z, Li D, Guo D, Ren Y, et al. Rates of mutation and host transmission for an Escherichia coli clone over 3 years. PLOS ONE. 2011;6:E26907.
79. Savini V, Catavitello C, Talia M, Manna A, Pompetti F, Favaro M, et al. Multidrug-resistant Escherichia fergusonii: a case of acute cystitis. J Clin Microbiol. 2008;46(4):1551-2.

80. David LA, Weil A, Ryan ET, Calderwood SB, Harris JB, Chowdhury F, et al. Gut microbial succession follows acute secretory diarrhea in humans. mBio. 2015;6(3):e00381.

\section{Publisher's Note}

Springer Nature remains neutral with regard to jurisdictional claims in published maps and institutional affiliations.
Ready to submit your research? Choose BMC and benefit from:

- fast, convenient online submission

- thorough peer review by experienced researchers in your field

- rapid publication on acceptance

- support for research data, including large and complex data types

- gold Open Access which fosters wider collaboration and increased citations

- maximum visibility for your research: over 100M website views per year

At BMC, research is always in progress.

Learn more biomedcentral.com/submissions 